\title{
ÉVALUATION DE L'ÂGE ET DE LA CROISSANCE DE L'ANGUILLE EUROPÉENNE (ANGUILLA ANGUILLA L.) EN MILIEU CONTINENTAL : MÉTHODOLOGIES, VALIDATION, APPLICATION EN MÉDITERRANÉE ET COMPARAISONS EN EUROPE.
}

\author{
J. PANFILI (1), Marie-Claude XIMÉNÈS (2)
}

(1) ORSTOM, Laboratoire de Sclérochronologie des Animaux Aquatiques, B.P. 70, 29280 PLOUZANÉ, France.

(2) CEMAGREF, B.P. 5095, 34033 MONTPELLIER Cedex, France.

\section{RÉSUMÉ}

L'âge individuel de l'anguille en milieu continental est toujours un paramètre difficile à évaluer. Actuellement, seule l'otolithométrie est utilisée. Une description rapide des techniques de préparation et d'observation des otolithes, de leurs avantages et de leurs inconvénients, est donnée : observation in toto, ponçage, lames minces, brûlage, coloration, microscopie électronique à balayage, analyse chimique et analyse d'images. Elles sont axées sur l'interprétation des marques de croissance saisonnières. Malgré la diversité de ces méthodes, le choix d'une méthode fiable et universelle est difficile. De plus, les applications doivent être examinées et validées en fonction de chaque population de cette espèce dont la plasticité adaptative est remarquable.

Les expériences de validation des estimations de l'âge (connaissance exacte des rythmes de dépôts de marques identifiées) à partir des otolithes sont peu nombreuses dans la littérature. Deux expériences de ce type sont présentées pour des populations méditerranéennes : une validation directe, par marquage individuel à la tétracycline dans un étang naturel de Camargue, et une validation indirecte, par observation de l'apparition marginale des marques de croissance au cours du temps pour des populations du Languedoc (étang saumâtre) et de Camargue (canal dulçaquicole). Ces expériences ont permis de déterminer précisément la séquence de formation des marques de croissance sur les otolithes ainsi que leur identification en fonction du mode de préparation. Une zone opaque large se forme principalement au printemps, une zone hyaline large se dépose pendant les mois estivaux, et une "ligne d'arrêt de croissance" chromophile correspond à la période hivernale. Ces marques sont donc utilisables pour estimer l'âge des anguilles en région méditerranéenne. Il existe cependant une très grande variabilité des structures de croissance, des rythmes de dépôt et de la morphométrie des otolithes. Cette variabilité est présente au niveau des individus de la même population mais aussi entre les populations. Ces résultats aboutissent à une notion d'écotype pour les otolithes d'anguille : l'écotype est en relation avec le milieu colonisé par l'individu et semble être fonction de la salinité et probablement du niveau trophique; il retrace aussi l'histoire individuelle.

Ces variabilités interviennent directement sur la croissance des individus. Les croissances individuelles dans les milieux méditerranéens saumâtre et dulçaquicole ont été mesurées et comparées entre elles et avec la littérature. La croissance en milieu saumâtre est nettement plus importante que celle en milieu d'eau douce.

Mots-clés : anguille, Anguilla anguilla, âge, croissance, otolithe, otolithométrie, validation, variabilité, région méditerranéenne, Europe. 


\title{
AGE AND GROWTH ESTIMATION \\ OF THE EUROPEAN EEL (ANGUILLA ANGUILLA L.) IN CONTINENTAL WATERS : METHODOLOGY, VALIDATION, APPLICATION IN MEDITERRANEAN AREA AND COMPARISONS IN EUROPE.
}

\begin{abstract}
The individual age of eels in the continental waters is still difficult to evaluate. The otolithometry is the only method used. A short description of the preparation techniques and otolith observations is given, with their advantages and disadvantages : whole observation, grinding, slices, burning-cracking, dyeing, scanning electron microscopy, chemical analysis and image analysis. All these methods are based on the interpretation of seasonal marks on the otoliths. In spite of the extreme diversity of the methods, the choice of one universal and reliable is difficult. Applications have to be validated for each population of this species which has a strong adaptative plasticity.
\end{abstract}

There is only few validation experiments of the age estimations of eels in the literature (knowledge of the timing of the mark deposition on otoliths). Such experiments are presented for two Mediterranean populations : a direct validation with tetracycline labelling in a natural pond in Camargue, and a semi-direct validation with the observation of the timing apparition of otolith marks in lagoons. These experiments permitted to determine a precise rythm of mark deposition and to identify which marks can be used to estimate the age. A large opaque zone appears during the spring, a large hyaline zone deposits in summer and a structural stainable discontinuity is led down each winter. These marks can be usefull for the age estimations of eels in the Mediterranean area. There is a great variability of the growth marks, of the rythms depositions and of the otolith morphometry. This variability is shown for individuals of the same or different populations. These results conduct to the think that there is some "otolith ecotypes": the different ecotypes are in relation with the environment and seems to depend on the salinity and probably on the trophic level ; it shows also the individual history.

This variability influences directly the individual growth. Growths in Mediterranean environments such as lagoons were measured and compared to those of the literature. The growth in brackish water environment is greater than those in freshwater environments.

Key-words : eel, Anguilla anguilla, age, growth, otolith, otolithometry, validation, variability, Mediterranean area, Europe.

\section{INTRODUCTION}

L'estimation de l'âge et de la croissance des anguilles concerne la plupart du temps la phase de la vie en milieu continental. L'âge individuel est évalué de façon classique à partir des marques enregistrées et conservées sur les tissus durs au cours de la vie des individus (sclérochronologie ${ }^{*}$ ). Cette dernière est particulièrement délicate à utiliser à cause de la biologie particulière de l'anguille et de la diversité des milieux qu'elle colonise, qui donnent des modalités de croissance très variables.

Seule l'otolithométrie est utilisée, car la scalimétrie n'est pas fiable en raison de l'apparition tardive des écailles et de leur accroissement non régulier (JELLYMAN, 1979). D'autre part, aucune étude sérieuse n'a été effectuée sur la croissance du squelette interne (squelettochronologie). Si l'on s'accorde pour utiliser les otolithes, les synthèses exhaustives les plus récentes n'ont pas permis de conclure sur le choix d'une méthode de préparation (VOLLESTAD et al., 1988 ; LECOMTE-FINIGER, 1992b). Des comparaisons méthodologiques précises ont conduit à des désaccords (MORIARTY et STEINMETZ,

\footnotetext{
* Voir BAGLINIÈRE et al. (1992) pour la définition des termes spécifiques à la sclérochronologie utilisés dans cet article et indiqués en italique dans le texte.
} 
1979), n'ont pas permis de faire un choix (MANN et BLACKBURN, 1991), ou sont contradictoires (VOLLESTAD, 1985 ; VOLLESTAD et JONSSON, 1988 ; VOLLESTAD et NAESJE, 1988 ; VOLLESTAD et al., 1988). Seules une intercalibration des méthodes de préparation des otolithes et une définition précise des marques de croissance qu'elles révèlent peuvent permettre de standardiser les lectures (MOUNAIX, 1992b; PANFILI, 1993). D'autre part, les données concernant l'âge des diverses populations sont à prendre avec une certaine réserve puisque très peu de validations* ont été entreprises pour cette espèce fréquentant pourtant des biotopes extrêmement variés. Certains essais de validation ont été infructueux (BOETIUS, 1985, 1986 ; DEKKER, 1986) ou peu convaincants (APRAHAMIAN, 1987 ; VOLLESTAD et NAESJE, 1988). L'expérience de marquage vital à la tétracycline de DEKKER (1986) s'est par exemple heurtée aux problèmes de recapture et aux difficultés d'observation des otolithes. Deux autres expériences de marquage à la tétracycline ont été réalisées en étang (MOUNAIX, 1992a ; PANFILI et al., 1992) et ont permis de mieux comprendre les rythmes de dépôt sur les otolithes.

En fait, la diversité des techniques d'examen des structures de croissance des otolithes et le manque de validation des résultats ne font qu'accroître l'incertitude de leur interprétation. II est d'ailleurs possible que ceci soit à l'origine de la variabilité observée dans la croissance des individus à l'échelle de la répartition géographique de l'anguille européenne (FONTENELLE, 1991 ; VOLLESTAD, 1992). Mais ces différences pourraient également être dues aux différences de conditions environnementales rencontrées par les individus ou même à la variabilité de croissance individuelle marquée chez cette espèce (VOLLESTAD, 1989, 1992 ; PANFILI et al., 1994).

Cet article tentera : (1) de présenter les diverses techniques de préparation des otolithes et les marques de croissance mises en évidence ; $(2)$ de montrer comment les marques saisonnières ${ }^{* \star}$ peuvent être utilisées pour estimer l'âge en milieu continental, en insistant sur deux expériences de validation des dépôts conduites en Méditerranéé ${ }^{\star * \star}$; et (3) de donner les applications pour les estimations de la croissance.

\section{OTOLITHOMÉTRIE}

\section{Mise en évidence et interprétation des structures pour estimer l'âge}

Des trois paires d'otolithes présentes dans l'oreille interne, seule la sagitta; le plus volumineux, est préparée pour l'otolithométrie (le terme "otolithe" sera donc équivalent de "sagitta"). Les techniques de préparation des otolithes d'anguilles citées dans la littérature sont très variées (fig. 1).

De nombreux auteurs ont ainsi utilisé l'observation «in toto" dans des bains éclaircissants tels que l'alcool (VOLLESTAD, 1985 ; VOLLESTAD et JONSSON, 1988 ; FERNANDEZ-DELGADO et al., 1989 ; GORDO et JORGE, 1991), la créosote (MANN et BLACKBURN, 1991), le xylène (HANSEN et EVERSOLE, 1984 ; ARIAS et DRAKE, 1985), le salicylate de méthyle (MACEWAN et HETCH, 1984), le benzoate de méthyle (XIMÉNÉS, 1986), l'essence de camomille (LECOMTE-FINIGER, 1985 ; MOUNAIX, 1992b), l'essence de romarin (PANFILI, 1993). L'observation de l'otolithe entier est faite le plus souvent en lumière réfléchie sur fond noir : les anneaux opaques ou hyalins sont ainsi décomptés pour estimer l'âge (fig. 2a). A partir de l'observation "in toto", les techniques de traitement numérique (analyse d'image) permettent d'accentuer les différences de densités optiques entre les zones sous forme de niveaux de gris, et de caractériser les anneaux opaques et hyalins par les maxima et les minima respectivement (PANFILI et al., 1990 ; PANFILI, 1993). Le comptage et les mesures des zones de croissance peuvent alors être réalisés

\footnotetext{
* La validation est la vérification expérimentale des hypothèses sur les scénarios de croissance des marques sur les pièces dures.

** Voir LECOMTE-FINIGER (présent numéro) pour l'utilisation des microstructures des otolithes (marques journalières) et l'évaluation de l'âge durant la phase de vie marine.

*** Les résultats présentés sont extraits principalement de PANFHLI (1993). Les sites expérimentaux sont localisés dans la région méditerranéenne française (une lagune languedocienne, un canal dulçaquicole et un étang camarguais). Ces résultats sont comparés et intégrés à ceux de la littérature.
} 


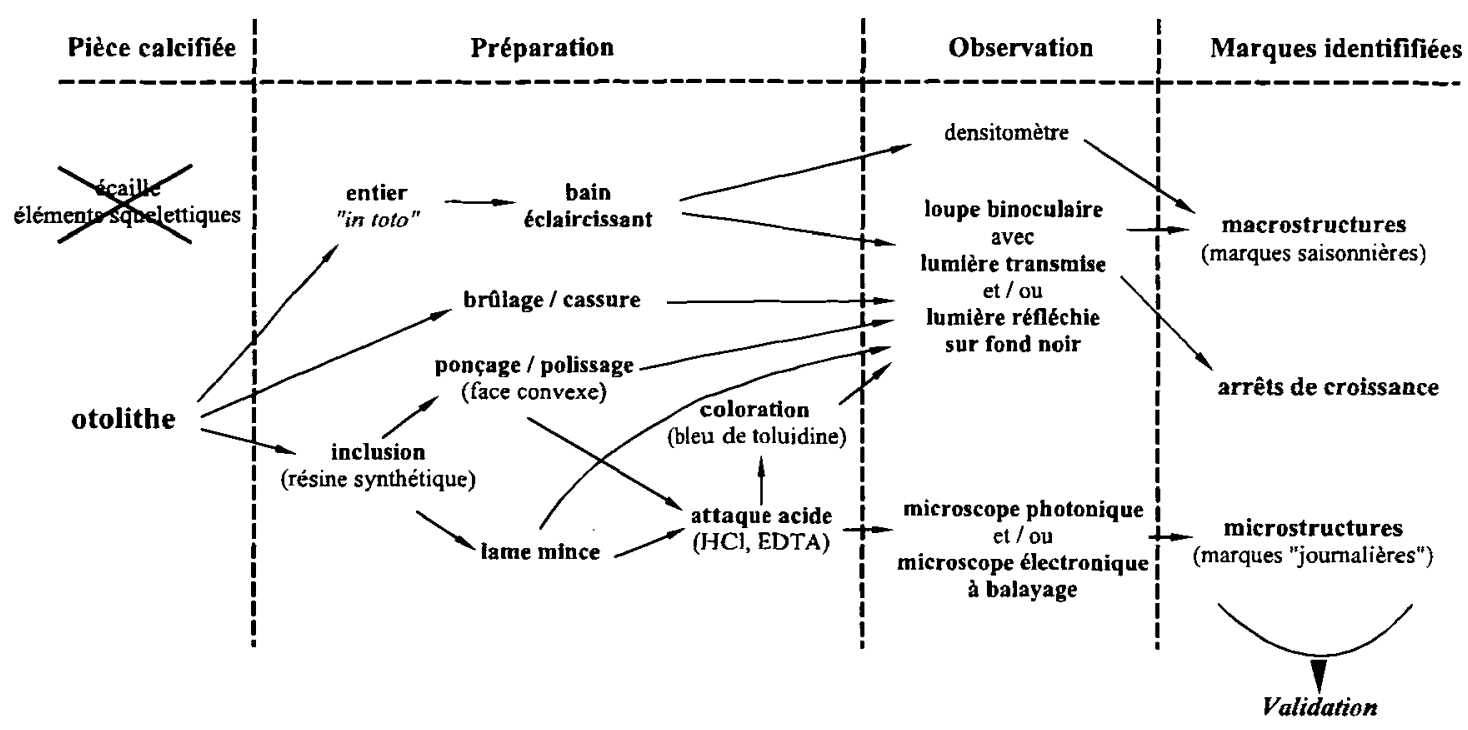

\section{Figure 1 : Différentes méthodes de préparation et d'observation des otolithes d'anguilles utilisées dans la littérature pour estimer l'âge.}

Figure 1 : Different methods of preparation and observation of eel otoliths used in the literature for the age estimation.

automatiquement (fig. 3). Les zones centrales de l'otolithe représentent la phase de vie marine et sont regroupées sous le terme de nucleus (figs. 2 et 3 ; et voir page 8 ). La croissance en milieu continental concerne donc les zones faisant suite au nucleus.

Le ponçage est souvent utilisé pour améliorer la lisibilité des marques de croissance en accentuant les contrastes entre les zones et en éliminant l'opacité générale. II peut nécessiter un enrobage préalable dans de la résine synthétique à cause de la très petite taille de l'otolithe (VOLLESTAD et al., 1988). Les plans de ponçage sont très variés (fig. 4) : le ponçage de la face interne (convexe) dans le plan sagittal est le plus commun (SINHA et JONES, 1967 ; MORIARTY et STEINMETZ, 1979 ; MACEWAN et HETCH, 1984 ; LECOMTE-FINIGER, 1985). L'obtention de lames minces selon le plan frontal (fig. 4) avait été recommandé par le ICES/EIFAC en 1979 puis utilisée dans diverses études (DEELDER, 1981 ; BERG, 1985 ; PAULOVITS et BIRO, 1986 ; VÉRO et al., 1986). Cette méthode semble maintenant abandonnée à cause de la difficulté de préparation et d'observation des structures de croissance.

La technique du brûlage/cassure («burning-cracking» en anglais) a été largement utilisée (MORIARTY, 1973, 1983 ; RASMUSSEN et THERKILDSEN, 1979 ; HU et TODD, 1981 ; BOETIUS, 1985 ; VOLLESTAD, 1985 ; APRAHAMIAN, 1986, 1987, 1988 ; DEKKER, 1987 ; VOLLESTAD et NAESJE, 1988 ; CHISNALL et HAYES, 1991 ; POOLE, 1991). Les zones les plus riches en protéines sont ainsi calcinées et dénombrées pour estimer l'âge. Pourtant la fiabilité de cette méthode a été remise en cause (VOLLESTAD, 1985) et la manipulation des otolithes calcinés est délicate (VOLLESTAD et al., 1988). Les études les plus récentes (MOUNAIX, 1992b; PANFILI, 1993) montrent que la durée de la calcination et le plan de cassure sont difficilement maîtrisables. L'utilisation de cette technique reste donc controversée.

La coloration de la surface poncée, polie, puis décalcifiée de l'otolithe révèle des lignes chromophiles plus ou moins intenses (fig. $2 \mathrm{~b}$ ). En général, la face convexe polie est décalcifiée avec du $\mathrm{HCl}$ (acide chlorhydrique) ou de l'EDTA (acide éthylène-diaminetétraacétique) et colorée avec du bleu de toluidine (ALBRECHTSEN, 1968 ; LIEW, 1974 ; 

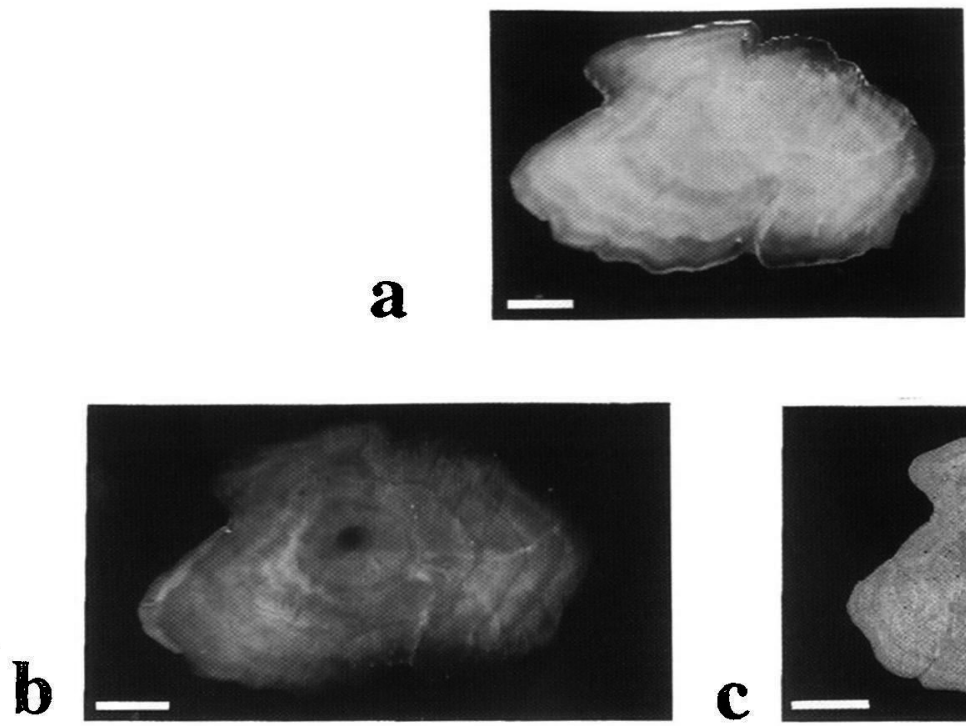

C
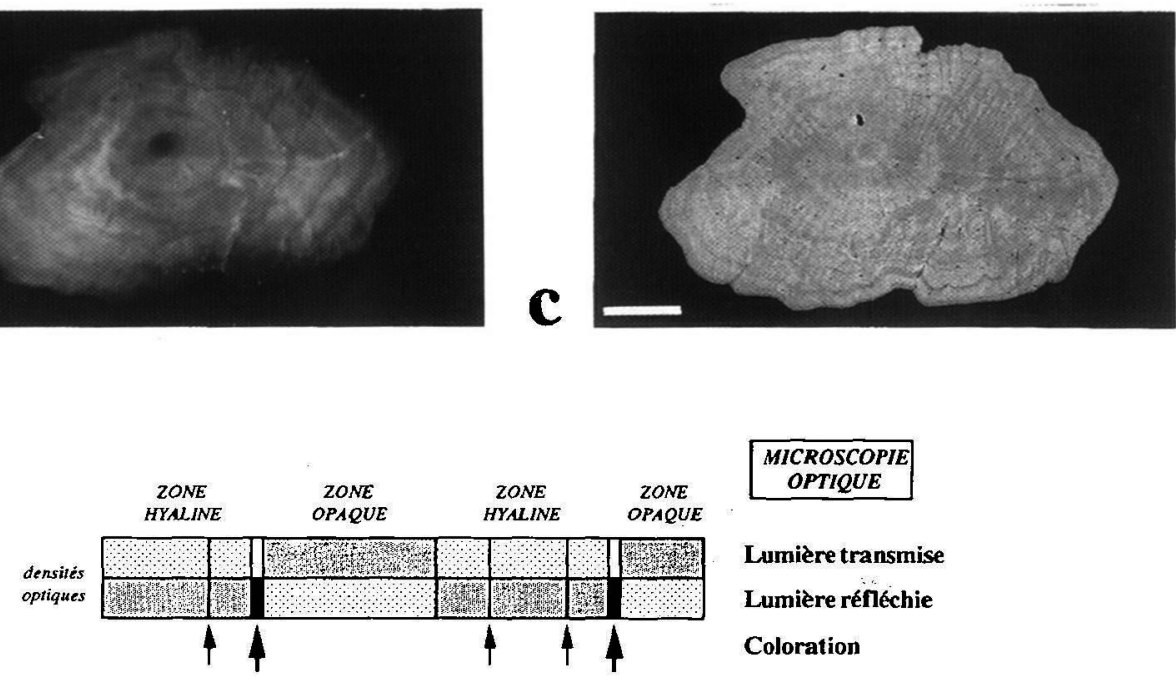

OPTIQUE

Lumière transmise

Lumière réfléchie

Coloration

MICROSCOPIE

ELECTRONIQUE

A BALAYAGE

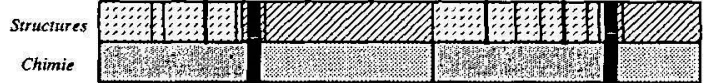

Image secondaire

Image rétrodiffuscé

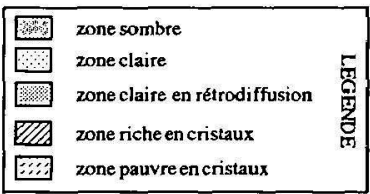

d

Figure 2 : Otolithe d'une anguille de $54,7 \mathrm{~cm}$ observé après diverses préparations. Barre $=\mathbf{5 0 0} \mathbf{\mu m}$.

a) Otolithe poncé en lumière réfléchie sur fond noir.

b) Otolithe coloré par le bleu de toluidine.

c) Image rétrodiffusée de l'otolithe en MEB (image chimique).

d) Schéma synthétique des zones de l'otolithe telles qu'elles sont révélées par plusieurs techniques de préparation. Les échelles relatives des zones de croissance ne sont pas respectées.

Figure 2 : Otolith of a $54.7 \mathrm{~cm}$ eel observed after different preparation techniques. Bar $=500 \mu \mathrm{m}$.

a) Ground otolith in reflected light against a dark background.

b) Otolith stained with toluidine blue.

c) Back-scattered image of the otolith in SEM (chemical image).

d) Diagram of the growth zones of the eel otolith as they appear with different preparation techniques. The relative scales of the growth zones are not respected. 

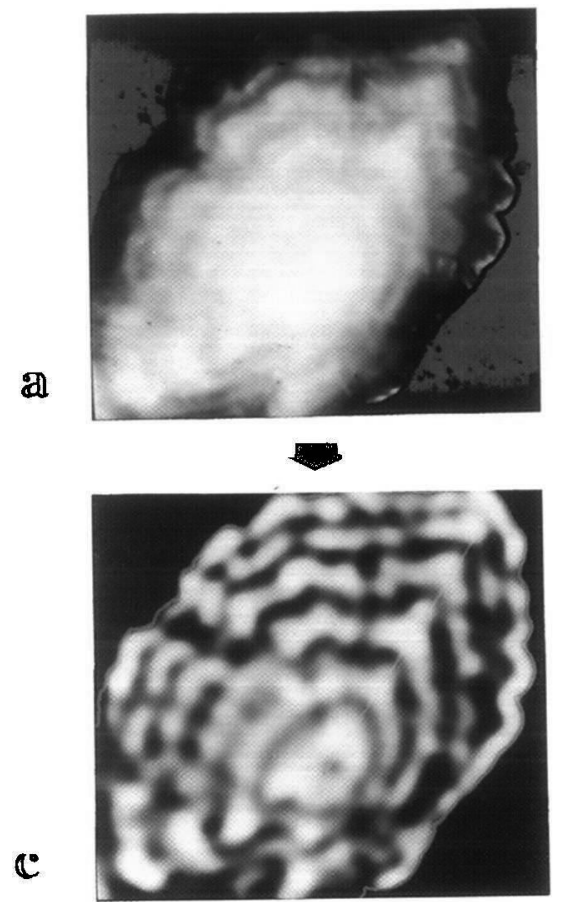
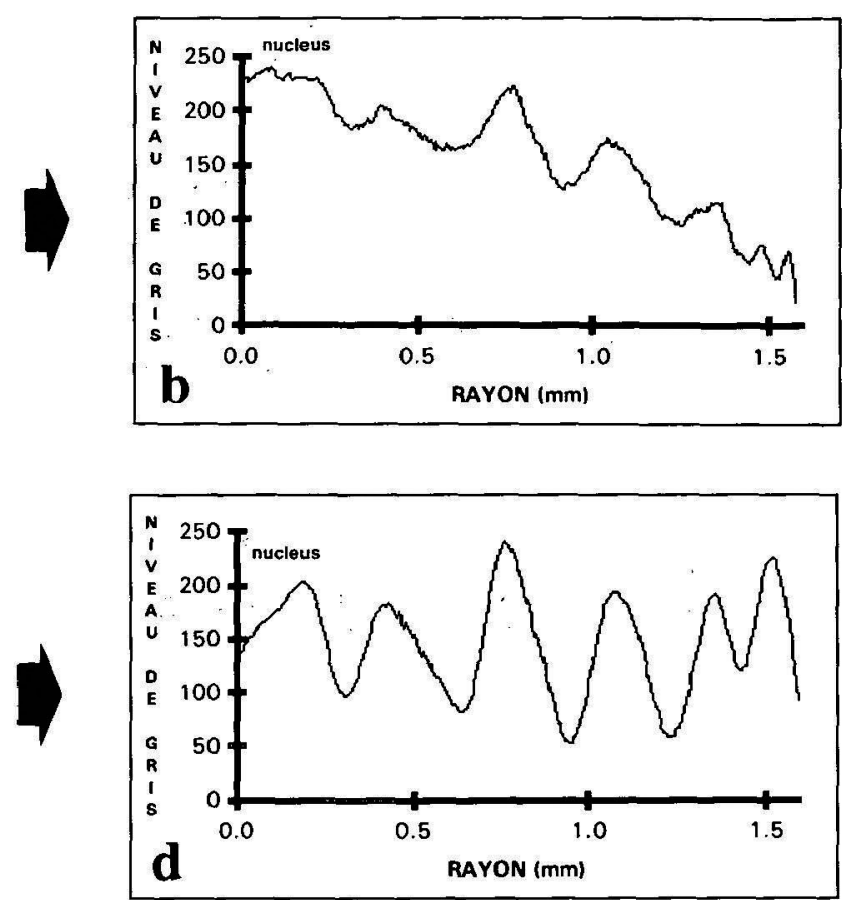

Figure 3 : Images d'un otolithe et de son profil avant et après traitement numérique en analyse d'image.

a) Image non traitée de l'otolithe en lumière réfléchie sur fond noir : son contour est peu précis ; le centre du nucleus n'est pas positionné ; les anneaux sont peu contrastés.

b) Profil acquis sur l'image non traitée de l'otolithe sur le rayon centre-bord postérieur (courbe de niveaux de gris, i.e. densités optiques, sur un axe de l'image) : la pente du profil est décroissante, rendant les anneaux du bord peu distincts ; la courbe est irrégulière et possède de nombreux artefacts.

c) Image du même otolithe après traitement, avec son contour et le rayon de lecture visibles en "overlay".

d) Profil de l'otolithe après traitement suivant le rayon de la figure précédente (3c). Les anneaux opaques sont caractérisés par les maxima et les anneaux hyalins par les minima.

Figure 3 : Otolith images and its profiles before and after numerical treatment in image analysis.

a) Non treated image of the otolith in reflected light against a dark background : the outline is not precise ; the nucleus centre is not marked; the zones are not underlined.

b) Non treated profile of the otolith on its anterior-posterior radius (grey level curve, i.e. optical densities, on the image axis) : the profile slope is decreasing, the marginal zones are then not distinct, the curve is irregular and shows numerous artefacts.

c) Image of the same otolith after numerical treatment with its outline and reading axis shown in overlay.

d) Otolith profile after treatment on the radius of the previous figure (3c). The opaque zones correspond to the maxima and the hyaline zones to the mimima. 
BENECH, 1975 ; CHARLON, 1975 ; NAGIEC et BAHNSAWY, 1990 ; MOUNAIX, 1992b ; PANFILI, 1993). Les durées de l'attaque acide et de la coloration sont de l'ordre de quelques minutes. Le bleu de toluidine se fixe préférentiellement sur les zones les plus décalcifiées : ce sont de fines discontinuités structurales ou lignes d'arrêt de croissance (LAC) qui correspondent aux périodes d'arrêt de la calcification de l'otolithe. Ces LAC chromophiles sont utilisées pour estimer l'âge. Cette technique a l'avantage de révéler de fines lignes concentriques bien individualisées dont les positions sont facilement mesurées.

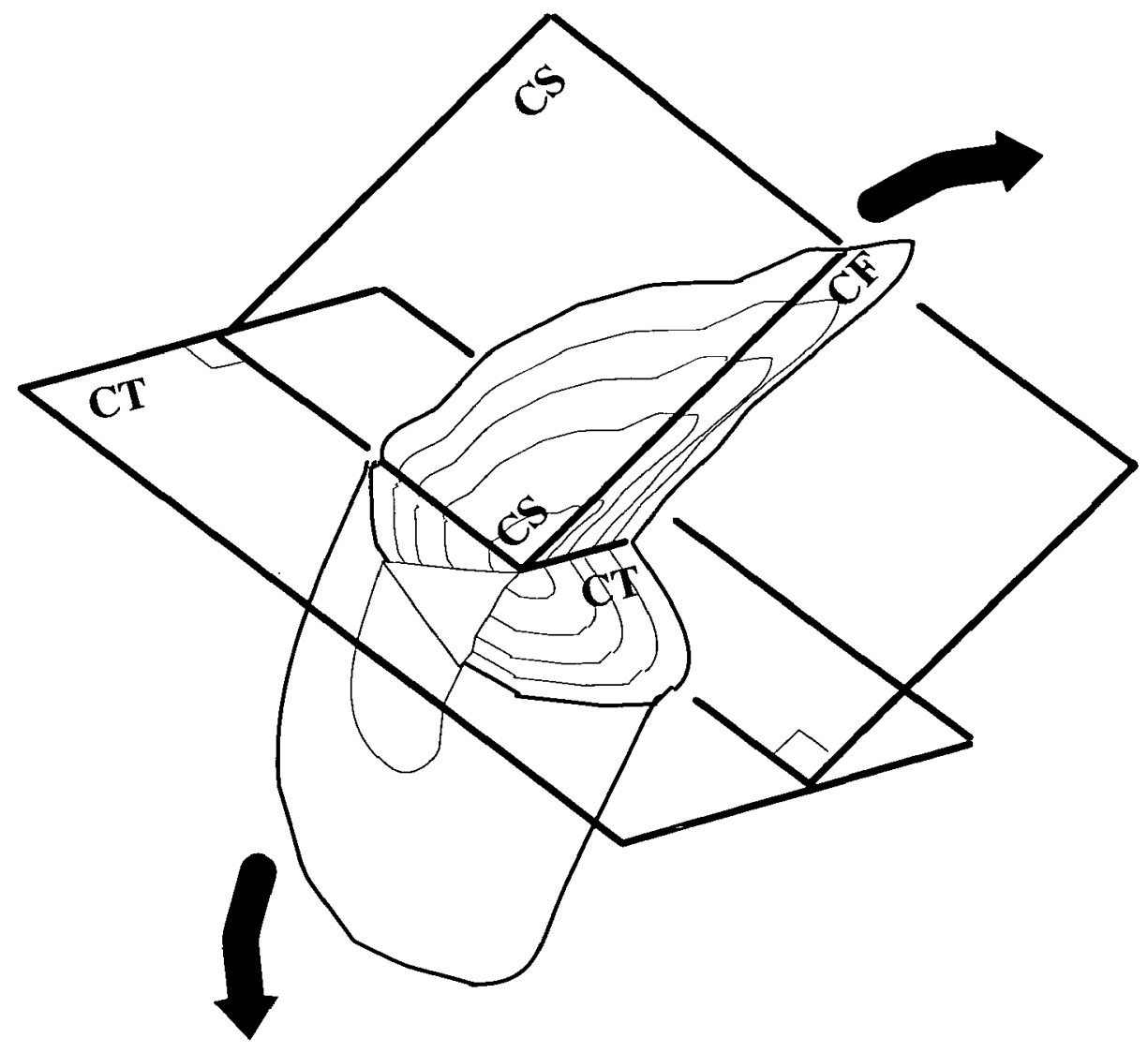

Figure 4 : Différents plans de ponçage de l'otolithe. CF, coupe frontale ; CS, coupe sagittale ; $\mathrm{CT}$, coupe transversale. Les flèches indiquent les directions de la concavité de l'otolithe.

Figure 4 : Different grinding planes of the otolith. CF, frontal plane ; CS, sagittal plane ; $\mathrm{CT}$, tranversal plane. The arrows show the concavity directions of the otolith.

Le microscope électronique à balayage (MEB) sert surtout à étudier l'ultrastructure de l'otolithe (LIEW, 1974 ; BERG, 1985 ; MICHAUD et al., 1988 ; LECOMTE-FINIGER, 1992a ; MOUNAIX, 1992b). Le MEB est aussi utilisé pour observer les microstructures et évaluer l'âge en jours (voir LECOMTE-FINIGER, ce numéro, pour revue). La structure de l'otolithe d'anguille est classique, constituée de cristaux d'aragonite inclus au sein d'une matrice protéique (LIEW, 1974 ; LECOMTE-FINIGER, 1992a). Les LAC sont visibles en MEB et peuvent être "simples" ou "composites" (MOUNAIX, 1992b). L'image rétrodiffusée de l'otolithe en MEB donne une image chimique de l'otolithe (fig. 2c), les zones les plus foncées étant les plus pauvres en calcium (PANFILI, 1993). 
Les essais d'intercalibration des méthodes de préparation des otolithes d'anguille montrent toute la difficulté à établir des critères simples d'identification des marques de croissance pour estimer l'âge (MOUNAIX, 1992b ; PANFILI, 1993). Le cas des otolithes issus de populations lagunaires méditerranéennes est singulier (fig. 2) : la largeur importante des zones de croissance facilite l'interprétation et les comparaisons (fig. $2 \mathrm{~d}$ ). Les LAC chromophiles se situent en bordure externe des zones hyalines larges, à la limite des zones opaques larges, voire à l'intérieur de ces dernières. La LAC peut se repérer plus ou moins facilement sur l'otolithe entier sous la forme d'un fin anneau très hyalin (laissant facilement passer la lumière). Les zones hyalines sont hypominéralisées, associées à des phases de croissance ralentie, et à l'inverse les zones opaques sont hyperminéralisées et associées à des phases de croissance active. Un certain nombre de LAC moins marquées (moins colorées et moins profondes en MEB) apparaissent au sein des zones hyalines. $\mathrm{Ce}$ genre de structures a engendré la notion de «zones surnuméraires» dans la littérature (DEELDER, 1976, 1981 ; VÉRO et al., 1986 ; LECOMTE-FINIGER, 1992a ; MOUNAIX, $1992 b)$. Cette notion peut être très subjective sans validation ou description très précise des marques de croissance. MOUNAIX (1992a, 1992b), ayant marqué des otolithes à la tétracycline pour une population dulçaquicole, estime que le MEB est absolument nécessaire pour discriminer les anneaux surnuméraires des "vrais" anneaux (LAC chromophiles). Pour les anguilles des lagunes méditerranéennes, la présence d'anneaux "surnuméraires" est plus rare dans les zones de croissance. La même observation a été faite par MOUNAIX (1992b) pour les anguilles estuariennes.

L'intercalibration des méthodes est bonne en ce qui concerne les zones du nucleus (figs. 2 et 5). Les observations des différents auteurs s'accordent (SINHA et JONES, 1967 ; LIEW, 1974 ; LEE, 1979 ; MICHAUD et al., 1988 ; LECOMTE-FINIGER et YAHYAOUI, 1989 ; NAGIEC et BAHNSAWY, 1990 ; LECOMTE-FINIGER, 1992a). La zone centrale du nucleus, hyaline et circulaire, est très chromophile, ainsi que la fine zone hyaline de transition l'entourant (fig. 2b). PANFILI et al. (1992) estiment que les zones hyalines et opaques sur les otolithes entiers ou les anneaux chromophiles peuvent être décomptés après cette zone de transition pour estimer l'âge ; une phase de validation est nécessaire pour confirmer ces affirmations.

\section{Variabilité et notion d'écotypes}

Dans les lagunes méditerranéennes, la première zone opaque qui fait suite au nucleus possède une épaisseur très variable pour les individus issus d'une même population (fig. 5). Cette zone se situe directement après la zone de transition marquant le passage à la vie continentale. Comme la période d'arrivée des civelles a lieu toute l'année, et s'étend surtout entre septembre et mai (FINIGER, 1976), l'épaisseur de la première zone opaque dépendrait de leur date d'arrivée. Cette zone serait donc formée entre l'hiver et le printemps et serait plus large pour les premiers arrivants. L'estimation de l'âge en milieu continental varie donc d'un individu à l'autre et peut être décalée de plusieurs mois si l'on fixe comme «date de naissance» la fin du printemps.

Les schémas de croissance des otolithes des anguilles d'une même population sont extrêmement variés (fig. 6). Dans l'étang de Mauguio en Languedoc et pour des anguilles de tailles semblables, la forme des otolithes, l'aspect et le nombre d'anneaux opaques et hyalins peuvent être totalement différents (fig. 6a). En fait, les otolithes provenant d'un milieu lagunaire ont des anneaux opaques et hyalins larges alors que ceux venant de milieux d'eau douce ont généralement des anneaux plus étroits. Cette notion d'écotype a été notée par MOUNAIX (1991, 1992b, et voir ce numéro pour revue). La technique de coloration accentue ces différences (fig. 6b). D'autres individus montrent des anneaux multiples (fig. 6c) ou des schémas très complexes (fig. $6 \mathrm{~d}$ ). II est cependant très difficile de définir l'appartenance à un écotype donné : par exemple pour les anguilles lagunaires, les possibilités de migrations d'un milieu à l'autre augmentent certainement la difficulté d'interprétation des schémas de croissance. Toutes ces variations entraînent un nombre d'échecs dans les lectures des otolithes dont les auteurs parlent peu souvent : PANFILI (1993) trouve $10 \%$ d'otolithes non interprétables pour une lagune méditerranéenne et $30 \%$ dans un canal dulçaquicole de Camargue. 

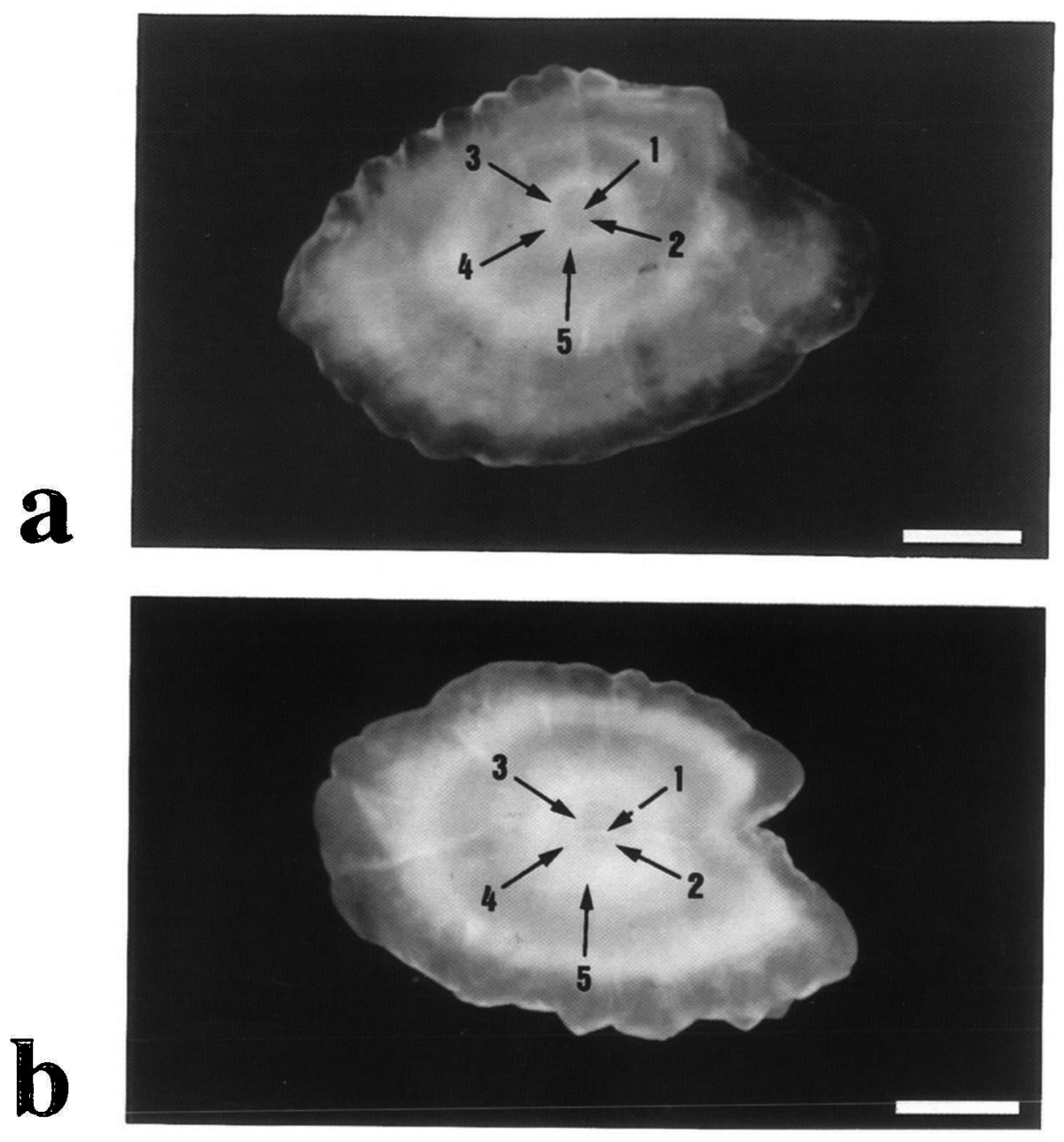

Figure 5 : Zones centrales de. l'otolithe d'anguille en lumière réfléchie sur fond noir. 1 , primordium (peu visible) ; 2 , centre hyalin ; 3 , zone opaque fine ; 4 , zone hyaline fine $=$ zone de transition (passage du milieu marin au milieu continental) ; 5 , zone opaque variable (première zone formée en milieu continental). $1+2+3+4=$ nucleus (vie marine). Barre $=500 \mu \mathrm{m}$.

a) Exemple d'otolithe avec une zone 5 étroite.

b) Exemple d'otolithe avec une zone 5 large.

Figure 5 : Central zones of the eel otolith in reflected light against a dark background. 1 , primordium (not clear) ; 2 , hyaline centre ; 3 , thin opaque zone ; 4 , thin hyaline zone $=$ transition zone (marine life to continental life) $; 5$, variable opaque zone. $1+2+3+4=$ nucleus (marine life). Bar $=500 \mu \mathrm{m}$.

a) Example of otolith with narrow zone 5 .

b) Example of otolith with wide zone 5.

\section{VALIDATION DES INTERPRÉTATIONS}

D'une façon générale, il est impossible d'aboutir à des estimations correctes de l'âge individuel sans avoir identifié précisément les marques de croissance et leur période de formation. C'est le but de la validation. Dans le cas de l'anguille, ce type d'étude a été très peu entrepris en regard de la diversité des milieux colonisés par cet animal. On peut noter des expériences de marquage vital dans trois milieux (DEKKER, 1986 ; MOUNAIX, 1992a ; PANFILI et al., 1992), de suivi d'apparitions de marques de croissance au cours du temps 

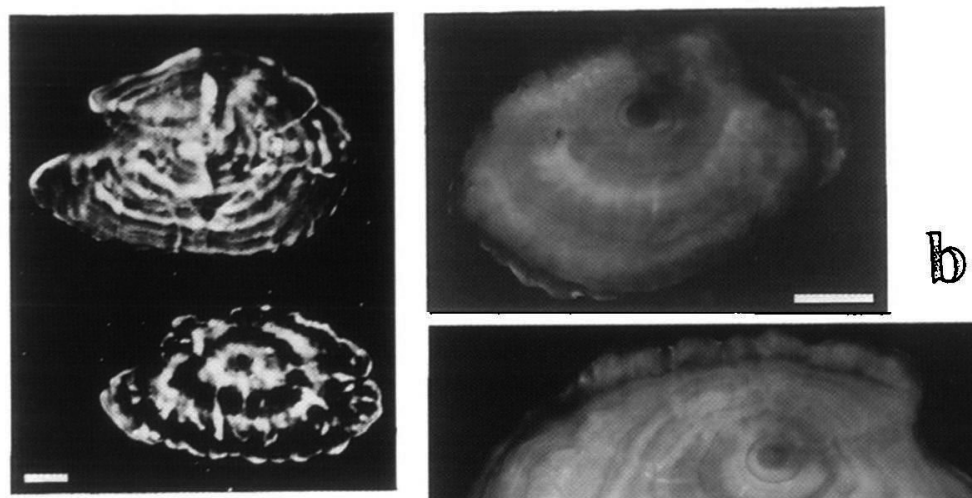

@

$\mathbb{C}$
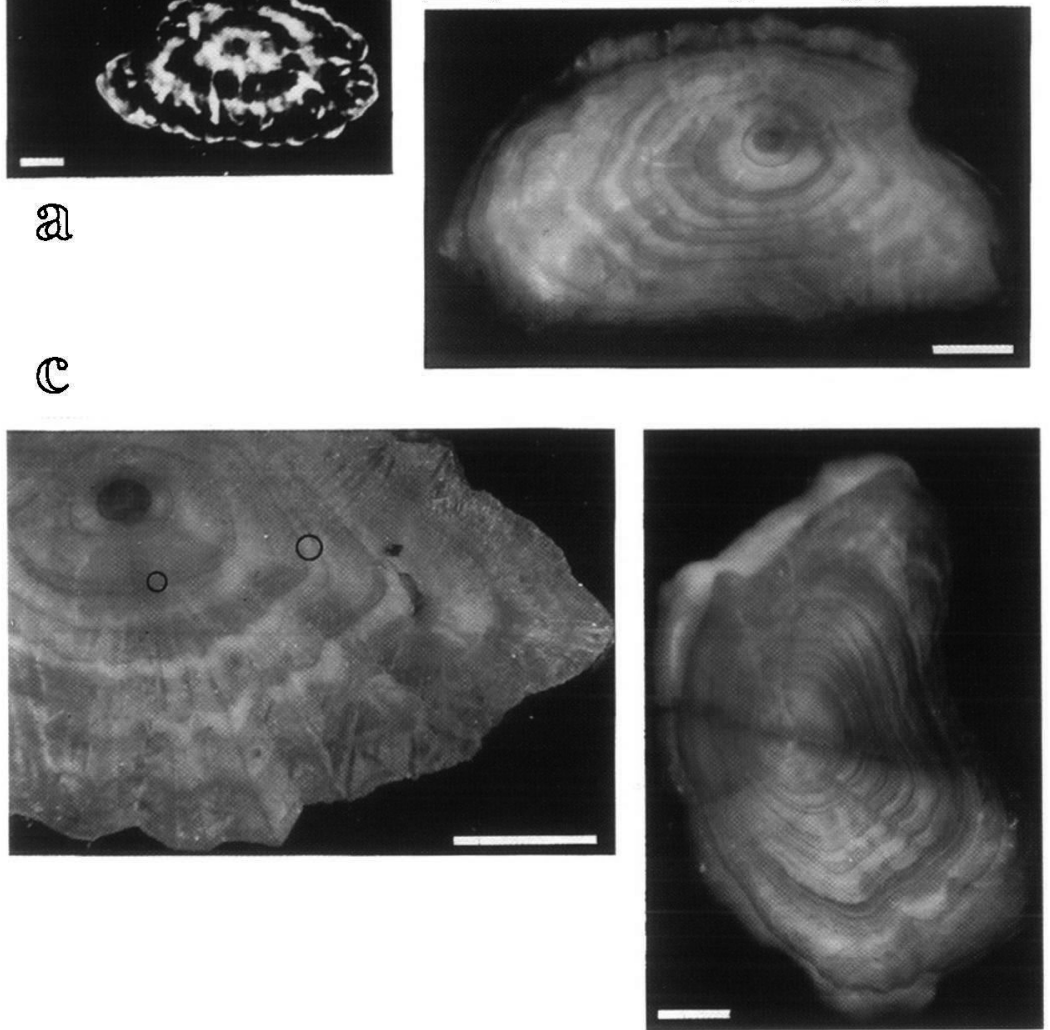

Q]

Figure 6 : Cas particuliers d'otolithes en lumière réfléchie sur fond noir. Barre $=500 \mu \mathrm{m}$. a) Otolithes d'anguilles pêchées à la même date (février 90) dans l'étang de Mauguio, France. L'otolithe en bas appartient à une anguille d'une longueur totale de $59,5 \mathrm{~cm}$ et celui du haut à une anguille de $58,0 \mathrm{~cm}$.

b) Otolithes colorés, à la même échelle, de deux anguilles capturées dans l'étang de Mauguio, France.

c) Otolithe coloré montrant des anneaux doubles (cercle).

d) Otolithe coloré.

Figure 6 : Particular examples of otoliths in reflected light against a dark background. Bar $=\mathbf{5 0 0} \mu \mathrm{m}$.

a) Otoliths of eels captured at the same date (February 90) in the Mauguio lagoon, France. The otolith below belongs to an eel of $59.5 \mathrm{~cm}$ total length and that above of an eel of $58.0 \mathrm{~cm}$.

b) Stained otoliths, at the same scale, of two eels caught in the Mauguio lagoon, France.

c) Stained otolith showing multiple rings (circle).

d) Stained otolith. 
(PANFILI, 1993) et de suivi de stocks de civelles au cours du temps (APRAHAMIAN, 1987 ; VOLLESTAD et NAESJE, 1988). Les résultats de ces diverses études ont été plus ou moins probants.

\section{Validation directe et application en Méditerranée}

La validation directe aboutit à la connaissance des scénarios de croissance à partir d'une pièce calcifiée d'un unique individu et grâce à la prise en compte d'un repère temporel précis par rapport aux marques de croissance. La méthode la plus utilisée est celle du marquage vital : elle consiste à fixer un fluoromarqueur sur les tissus durs à une date donnée et à l'observer rétrospectivement après une phase de croissance d'une certaine durée. Cette technique est utilisée depuis longtemps (WEBER et RIGWAY, 1967 ; MEUNIER, 1974 ; MEUNIER et BOIVIN, 1974 ; BEAMISH et MCFARLANE, 1983, 1987 ; inter alia). Dans le cas de l'anguille, la tétracycline (DEKKER, 1986 ; PANFILI et al., 1992) et la fluorescéine (MOUNAIX, 1992a) ont été injectées comme marqueurs vitaux. Ces derniers sont décelables a posteriori sous un éclairage épifluorescent.

Le très faible taux de recapture d'anguilles marquées de DEKKER (1986), dans un étang aux Pays-Bas, ne lui a pas permis d'identifier des marques précises sur les otolithes pouvant servir à l'estimation de l'âge. Par contre, les travaux de MOUNAIX (1992a) pour une population marquée dans un étang de l'ouest de la France et suivie pendant deux ans après le marquage ont conduit à une définition précise de la chronologie de formation des marques. Le marquage a une action calciotraumatique. D'après cet auteur, seul le MEB permet de distinguer les véritables $L A C$ formées chaque hiver, chromophiles par ailleurs, d'autres $L A C$ non annuelles. Ces discontinuités annuelles des otolithes ont une structure complexe et sont visibles au printemps, mais seraient dues à un ralentissement hivernal de la croissance.

L'expérimentation de PANFILI et al. (1992) a été conduite dans un étang de Camargue dans le but de valider les estimations de l'âge pour les populations méditerranéennes. Deux lots d'anguilles ont été marqués à la tétracycline et déversés au printemps et à l'automne 1989. Des captures régulières jusqu'à la fin du printemp 1990 ont permis de suivre l'apparition des zones de croissance des. otolithes après la marque de tétracycline et selon différentes méthodes de préparation (fig. 7). Le schéma de formation des zones diffère quelque peu de celui proposé par différents auteurs qui estiment que la zone hyaline se dépose en hiver et que la zone opaque est estivale (SINHA et JONES, 1967 ; LIEW, 1974 ; LECOMTE-FINIGER, 1985). En région méditerranéenne il se dépose chaque année une zone opaque et une zone hyaline larges et plus ou moins homogènes (fig. 7). La zone opaque apparaît surtout au printemps mais peut commencer à se former dès l'automne. La zone hyaline se dépose au cours de l'été. L'arrêt ou le ralentissement de la croissance se manifeste sous la forme d'une LAC chromophile en hiver et se situe en limite ou à l'intérieur de la zone opaque.

\section{Validation semi-directe et application en Méditerranée}

La validation semi-directe nécessite une observation qualitative (aspect hyalin ou opaque du bord) ou quantitative (mesures des zones marginales) des marques de croissance sur un grand nombre d'individus prélevés régulièrement au cours du temps. La méthode consiste à observer la bordure de l'otolithe tout en sélectionnant une marque donnée et à suivre sa formation dans le temps pour la population échantillonnée. La plupart des études réalisées sur l'anguille présente des résultats qualitatifs sans valeurs moyennes (SINHA et JONES, 1967 ; LEE, 1979 ; LECOMTE-FINIGER, 1985). Les résultats quantitatifs apportés par FERNANDEZ-DELGADO et al. (1989) pour un milieu estuarien et par PANFILI (1993) pour une lagune et un canal dulçaquicole méditerranéens sont proches. Cette dernière étude montre clairement un cycle annuel de formation des zones opaques et hyalines (fig. 8) : le dépôt de la zone opaque peut débuter dès l'automne mais a lieu principalement au printemps et celui de la zone hyaline se déroule pendant l'été. Les précisions sur les dates de formation (intervalles de temps) ne sont pas très bonnes et il existe un certain décalage entre le milieu lagunaire (fig. $8 \mathrm{a}$ ) et le milieu dulçaquicole (fig. $8 \mathrm{~b}$ ). Les variabilités individuelles sont importantes et un certain pourcentage d'otolithes possède un bord qui n'est pas facilement interprétable (fig. 8). D'ailleurs on n'observe jamais $100 \%$ de bords opaques, car l'opacité 


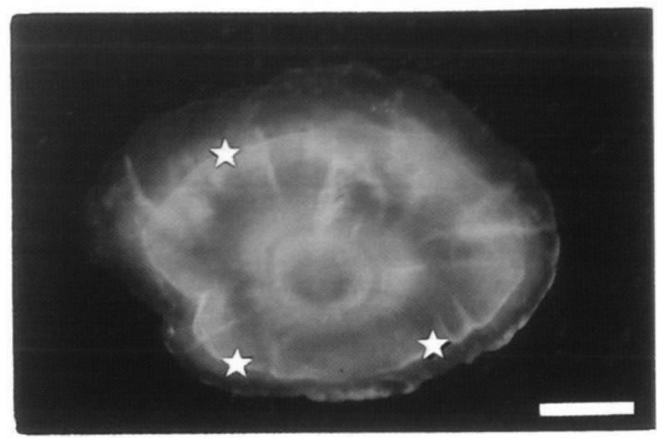

Q
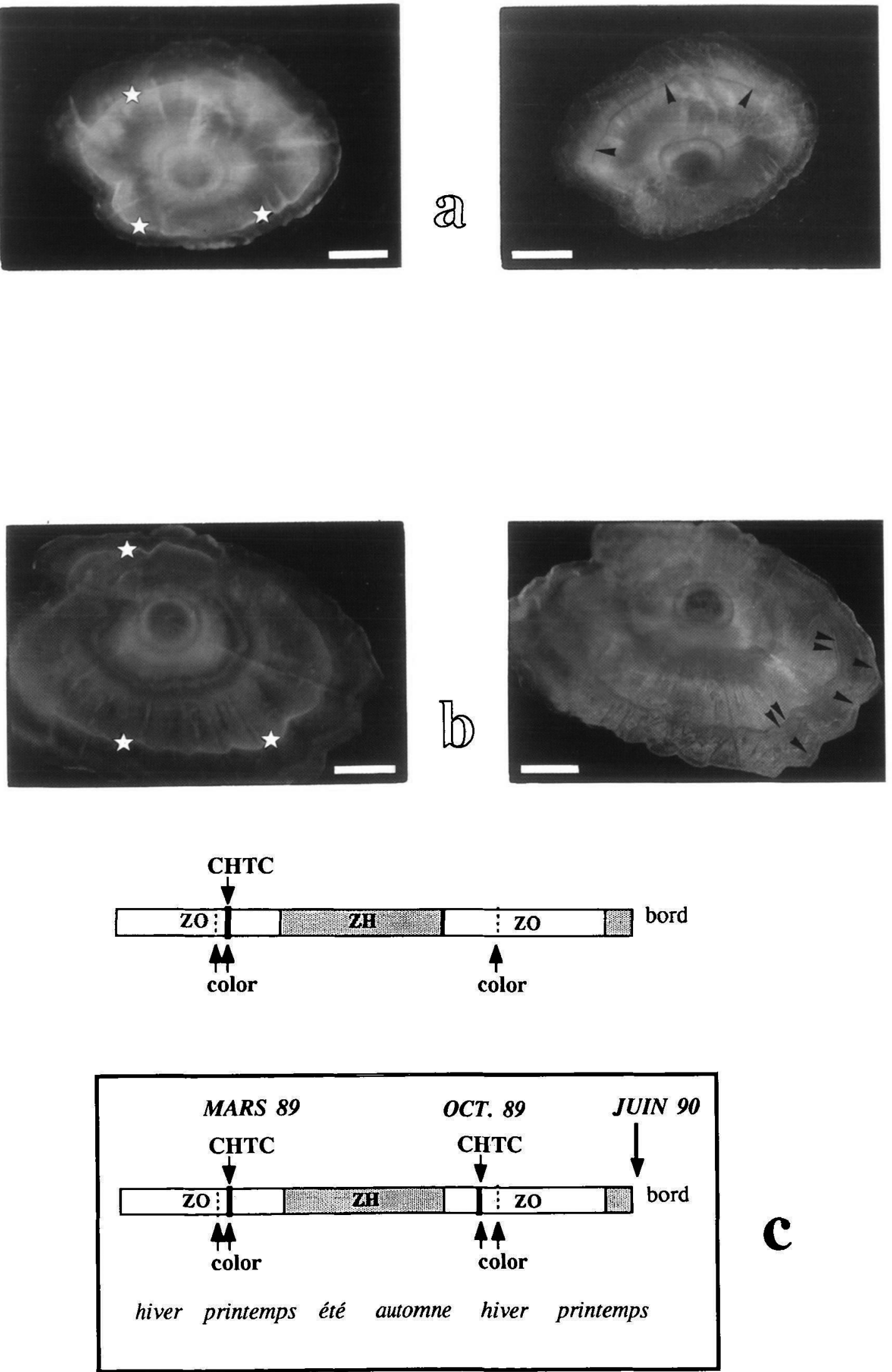

c

Figure 7 
Figure 7 : Otolithes marqués à la tétracycline et séquence de formation des zones de croissance. Otolithes en lumière réfléchie sur fond noir avec un éclairage en épifluorescence UV, sans coloration (gauche) et avec coloration (droite), et schémas interprétatifs des zones entre la marque de CHTC et le bord. Barre $=300 \mu \mathrm{m}$. Les schémas ne tiennent pas compte des échelles relatives de zones. CHTC, marque de tétracycline $(\star)$; color, anneau coloré ; ZH, zone hyaline ; ZO, zone opaque. (modifié d'après PANFILI et al., 1992).

a) Anguille marquée en mars 89 et pêchée en octobre 89.

b) Anguille marquée en mars 89 et pêchée en juin 90 .

c) Schéma synthétique des zones sur l'otolithe d'anguille après les différentes étapes de marquage et de préparation par coloration.

Figure 7 : Otoliths labelled with tetracycline and formation of the growth zones. Otoliths in reflected light against a dark background with an epifluorescent UV light, without staining (left) or with staining (right), and interpretative diagrams of the zones between the CHTC mark and the edge. Bar $=300$ $\mu \mathrm{m}$. The diagrams do not show the relative scales of the zones. CHTC, tetracycline mark $(\star)$; color, stained ring ; $\mathrm{ZH}$, hyaline zone; $\mathrm{ZO}$, opaque zone. (modified from PANFILI et al., 1992).

a) Eel marked in March 89 and captured in October 89.

b) Eel marked in March 89 and captured in June 90.

c) Diagram of the growth zones of the eel otolith after different steps of marking and preparations. 

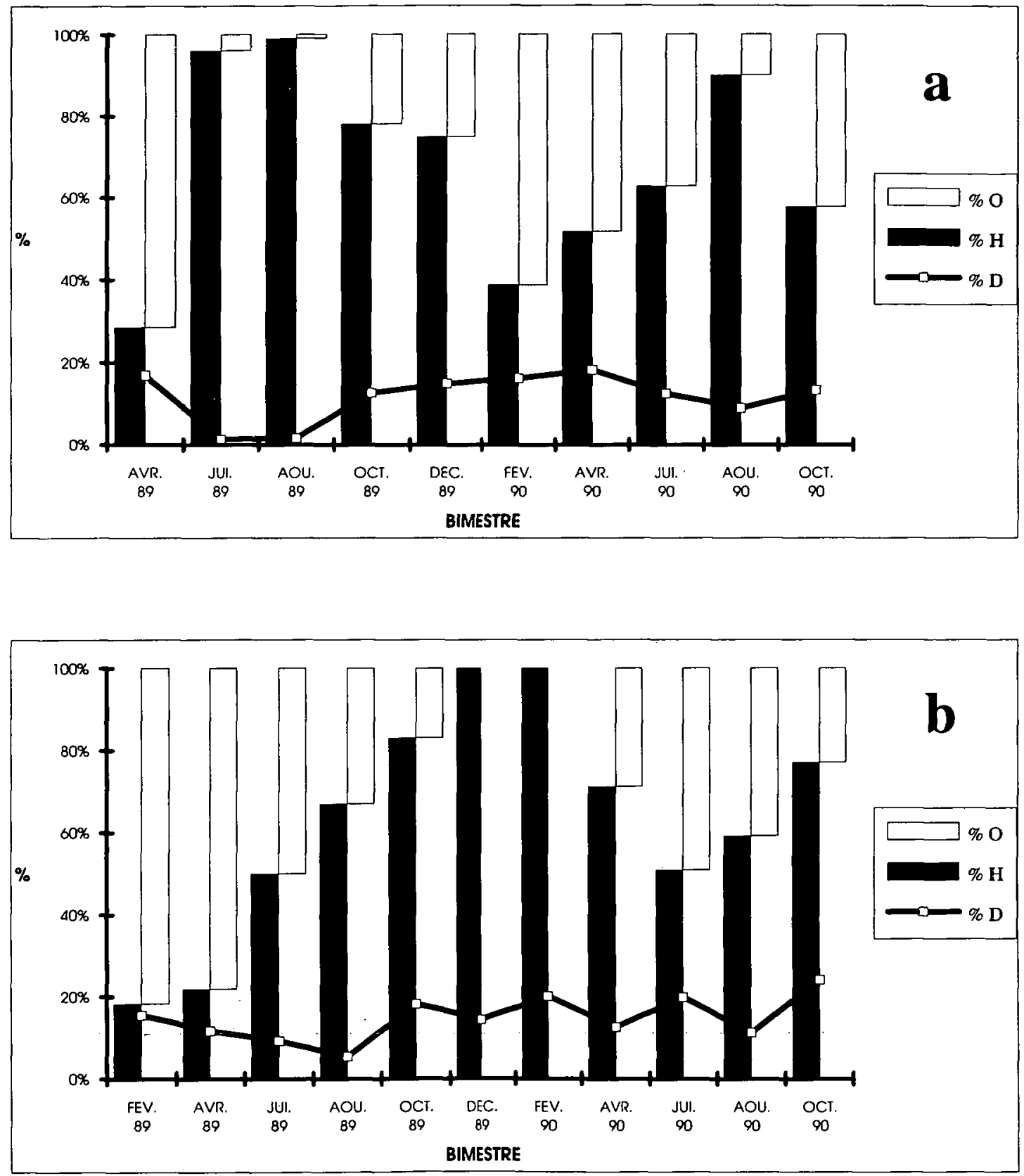

Figure 8 : Évolution du pourcentage de bords opaques ( $\%$ 0), de bords hyalins (\% $\mathrm{H})$, et de la difficulté à interpréter le bord $(\% \mathrm{D})$ en fonction du temps pour l'étang de Mauguio (a) et le canal du Fumemorte en Camargue (b) dans la région méditerranéenne française.

Figure 8 : Evolution of the percentage of opaque edges $(\% 0)$, hyalin edges $(\% H)$, and difficulty to interprate the edge $(\% \mathrm{D})$ during the time in the lagoon of Mauguio (a) and the canal of Fumemorte in Camargue (b) in the French Mediterranean area. 
marginale n'est visible que si la zone opaque est déjà bien formée. Ce type de validation nécessite de travailler sur un cycle supérieur à une année pour mettre en évidence des phénomènes cycliques variables entre les années et entre les individus. Ces résultats correspondent avec ceux obtenus avec les techniques de marquage vital (PANFILI et al., 1992 ; et paragraphe précédent).

\section{Paramètres du milieu influençant les dépôts}

L'évolution temporelle de l'apparition des zones de croissance des otolithes est en étroite relation avec l'évolution de certains paramètres physicochimiques du milieu (fig. 9) : la température et la salinité jouent un rôle dans le cas de la lagune de Mauguio (PANFILI, 1993). L'analyse est délicate puisque toutes ces variables sont liées entre elles. Les températures optimales de croissance des anguilles en élevage sont de l'ordre de 22 à $26^{\circ} \mathrm{C}$ (KUHLMAN, 1976, 1979 ; SADLER, 1979). En lagune méditerranéenne la température de l'eau dépasse $22^{\circ} \mathrm{C}$ pendant une longue période, de mai à septembre (fig. 9). Donc pendant les mois estivaux, synonymes de dépôts hyalins sur les otolithes, la température du milieu peut être considérée comme optimale pour la croissance. Aux époques où la température fléchit (printemps et automne), la calcification est modifiée et les zones opaques apparaissent. Dans ce même milieu, les températures inférieures à $10^{\circ} \mathrm{C}$ se rencontrent de novembre à février ; en dessous de ce seuil, les anguilles se nourrissent peu ou pas (HARALSTAD, 1984). C'est à ce moment que les LAC chromophiles se mettent en place. La température agit certainement de manière indirecte en influençant d'une part la disponibilité alimentaire et d'autre part le comportement alimentaire et l'activité des individus, en ayant une répercussion directe sur la croissance. La salinité semble être un facteur non négligeable pour le dépôt des zones de croissance (fig. 9), à la fois en termes de niveau et de variations. Cette observation rejoint les hypothèses de la présence d'écotypes d'otolithes en fonction des milieux (voir MOUNAIX, ce numéro).

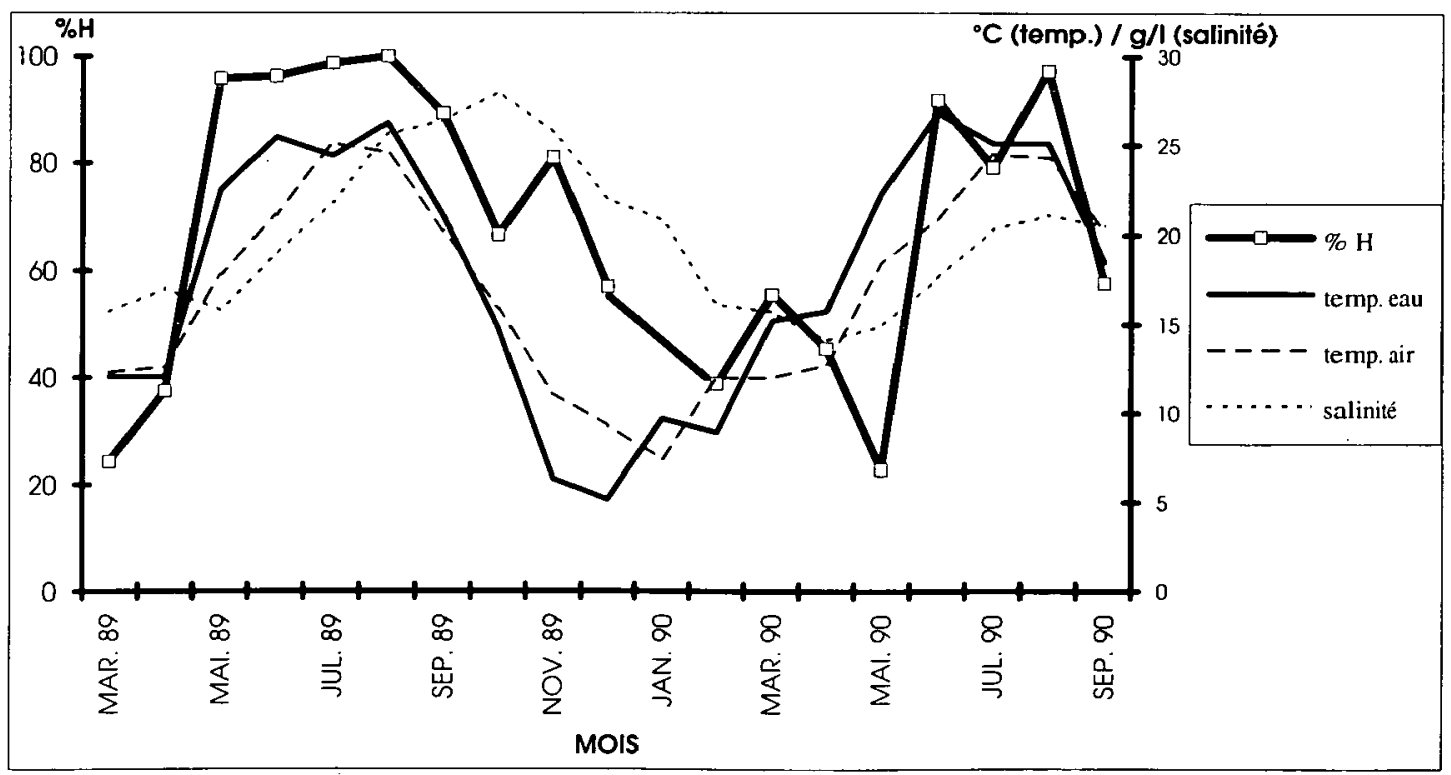

Figure 9 : Évolution mensuelle du pourcentage de bords hyalins $(\% \mathrm{H})$ et de quelques variables du milieu (temp. air, température de l'air ; temp. eau, température de l'eau ; salinité) dans l'étang de Mauguio en France.

Figure 9 : Monthly evolution of the percentage of hyalin edges $(\% \mathrm{H})$ and of some environmental variables (temp. air, air temperature; temp. eau, water temperature; salinité, salinity) in the Mauguio lagoon, France. 


\section{APPLICATION POUR LA CROISSANCE}

\section{Otolithes et estimations de l'âge}

S'il existe une différence pour les schémas d'apparition des zones de croissance entre les lagunes méditerranéennes et les autres milieux étudiés, ceci n'a pas d'incidence sur la détermination de l'âge en milieu continental. En effet, après la zone opaque périnucléaire qui correspond à la phase de recrutement, la somme d'une zone hyaline et d'une zone opaque correspond bien à un cycle annuel, et une LAC chromophile est formée chaque hiver. II est cependant nécessaire de valider les estimations pour chaque population étudiée puisque les méthodes utilisées ne sont pas obligatoirement les mêmes et doivent être adaptées au cas par cas.

Finalement, les estimations de l'âge à partir des otolithes de l'anguille européenne peuvent s'avérer fiables :

- pour les populations du bassin versant de la Vilaine en France (MOUNAIX, 1992a), où l'âge est estimé en dénombrant des discontinuités annuelles dont la structure composite est uniquement visible en MEB ;

- pour les populations des lagunes méditerranéennes en France (PANFILI et al., 1992), où l'âge est estimé en comptant les larges zones opaques faisant suite au nucleus et déposées annuellement, ou les LAC chromophiles révélées en coloration ; dans ce cas, à la première zone opaque dénombrée, l'individu pourra avoir entre 1 an et 1 an et demi de vie continentale à cause de la période de recrutement étalée dans le temps ;

- dans une moindre mesure pour les populations des étangs des Pays-Bas (VOLLESTAD et NAESJE, 1988) où l'âge est estimé en dénombrant les anneaux calcinés des otolithes après brûlage et cassure; il faut noter une certaine contradiction de cette étude avec la précédente de VOLLESTAD (1985);

- et dans une moindre mesure également, pour une population d'un étang aux Pays-Bas (DEKKER, 1987) en utilisant les anneaux relevés par la technique du brûlage.

Si l'on tient compte des variabilités intra- et inter-populationnelles sur les otolithes, les disparités de croissance peuvent être grandes. Ces observations ne sont pas surprenantes dans le cas de ce poisson ubiquiste présent dans tous les milieux continentaux à l'échelle européenne. II semble que les variations de croissance puissent être sous la dépendance d'un gradient latitudinal (VOLLESTAD, 1989), mais ce facteur ne peut à lui seul expliquer la diversité des situations présentées dans la littérature (FONTENELLE, 1991). L'hétérogénéité des méthodologies utilisées pour estimer l'âge peut également être mise en cause (FONTENELLE, 1991). La technique du brûlage est certainement la plus controversée et c'est pourtant l'une des plus utilisées et la seule qui donne des estimations supérieures à 20 ans (MORIARTY, 1983 ; POOLE, 1991). Il existe peu de comparaisons dans un même biotope en utilisant différentes méthodes ou des comparaisons entre des biotopes avec une même méthode. La correspondance de tous ces travaux "mono-biotopes" est donc délicate car chaque auteur utilise son propre mode de lecture des otolithes. La confrontation des lectures d'âge entre auteurs s'est d'ailleurs heurtée à de nombreux problèmes (MORIARTY et STEINMETZ, 1979), de même que celle pour un lecteur utilisant plusieurs méthodes pour une seule population (VOLLESTAD, 1985). Dans les comparaisons qui suivront, toutes les études seront considérées sans toutefois tenir compte des possibilités d'erreur ou du manque de validation des estimations d'âges.

\section{Rétrocalcul}

Le rétrocalcul est une méthode mathématique permettant de calculer la taille d'un poisson à une date antérieure, grâce aux mesures des marques de croissance précises sur les otolithes (voir FRANCIS, 1990, pour revue). Cette méthode a été beaucoup utilisée dans le cas de l'anguille, particulièrement lorsque les effectifs sont faibles. Le principe est (1) de calculer la relation liant la taille du poisson à celle de son otolithe (rayon par exemple), puis (2) de calculer la taille du poisson à la nième marque, connaissant la taille du poisson à la capture, le rayon de l'otolithe à la capture et le rayon de l'otolithe à la nième marque. Les résultats du rétrocalcul doivent toujours être interprétés avec attention car les méthodes mathématiques sont nombreuses (FRANCIS, 1990) et les possibilités de biais peuvent être non négligeables (SMALL et TAYLOR, 1987 ; CAMPANA, 1990 ; RICKER, 1992). 
Les relations entre la taille des anguilles et une mesure sur les otolithes sont toujours de type linéaire (Tableau I). Même si le type de relation est identique, les valeurs des paramètres (pente et ordonnée à l'origine) sont très différentes en comparant plusieurs biotopes à travers l'Europe (Tableau I). Ces relations, qui interviennent dans le rétrocalcul, devront donc être établies précisément pour chaque population étudiée. Ceci donne encore plus d'importance à la notion d'écotypes reconnus à partir des structures de croissance des otolithes. Les relations morphométriques entre la taille du poisson et celle de son otolithe sont aussi utilisées pour caractériser les différents stocks d'une même espèce (DAWSON, 1986 ; HOPKINS, 1986 ; MACEINA et MURPHY, 1989 ; MESSIEH et al., 1989 ; CASTONGUAY et al., 1991 ; FRIEDLAND et REDDIN, 1994 ; inter alia). Dans le cas de l'anguille la discrimination est particulièrement délicate à cause des possibilités de migrations d'un milieu à l'autre.

Tableau I : Relations entre la taille des anguilles jaunes (LT, longueur totale) et le rayon. (R) ou le diamètre (D) de l'otolithe selon différents auteurs et différents milieux européens. Régressions linéaires du type : $L T=a(R$ ou $D)+b . D$, diamètre de l'otolithe entre l'extrémité du rostre antérieur et le bord postérieur ; $n$, effectif ; Rp, rayon postérieur de l'otolithe (distance entre le centre du nucleus et le bord postérieur) ; R1, distance entre le premier anneau faisant suite au nucleus et le bord postérieur ; r2, coefficient de détermination ; tailles, gamme de tailles utilisée.

Table 1 : Relationships between the size of yellow eels (LT, total length) and the radius (R) or the diameter (D) of the otolith from different authors and different European areas. Linear regressions : $L T=a(R$ or $D)+b$. $D$, otolith diameter between the anterior rostrum and the posterior edge; $n$, number of individuals; $\mathbf{R p}$, otolith posterior radius (between the nucleus centre and the posterior edge) ; R1, distance between the first ring after the nucleus and the posterior edge; $r 2$, coefficient of determination ; tailles, range of sizes used.

\begin{tabular}{|c|c|c|c|c|c|c|c|c|c|}
\hline auteurs & milieu & pays & $\begin{array}{l}\text { tailles } \\
(\mathrm{cm})\end{array}$ & $\begin{array}{l}\text { mesures } \\
\text { otolithe }\end{array}$ & méthode & $\mathrm{n}$ & a & b & $r^{2}(\%)$ \\
\hline \multicolumn{10}{|l|}{ HETÉ̀ $\mathrm{H}$ et } \\
\hline AP̈ṔELBAUM (1977) & élevage & Allemagne & $9-61$ & $\mathrm{Rp}$ & entier & 51 & 25,0 & 14,5 & 85 \\
\hline ROSSI et VILANI (1980) & lagune & Italie & $33-75$ & $\mathrm{Rp}$ & entier & 444 & 38,2 & 0,40 & 67 \\
\hline $\begin{array}{l}\text { VOLLESTAB et } \\
\text { JONSSON (1988) }\end{array}$ & rivière & Norvège & $6 \cdot 26$ & $R p$ & entier & 373 & 27,0 & 15,1 & 84 \\
\hline $\begin{array}{l}\text { FERNAN̂NOEZZ-DELGADO } \\
\text { et al. }(1989)\end{array}$ & estuaire & Espagne & $5-60$ & $\mathrm{Rp}$ & entier & 412 & 26,4 & $-0,32$ & 95 \\
\hline $\begin{array}{l}\text { NAGIEC et } \\
\text { BAHNSAWY (1990) }\end{array}$ & lac & Pologne & $30 \cdot 100$ & $\mathrm{R} 1$ & coloré & 37 & 14,1 & 4,80 & 66 \\
\hline MOUNAIX (1992b) & estuaire & France & $10-75$ & $D$ & coloré & 60 & 0,20 & $-73,9$ & 81 \\
\hline MOUNAIX (1992b) & fleuve & France & $10-75$ & $\mathrm{D}$ & coloré & 82 & 0,17 & $-60,3$ & 52 \\
\hline PANFILI (1993) & lagune & France & $15 \cdot 65$ & $\mathrm{Rp}$ & entier & 895 & 29,8 & 1,84 & 74 \\
\hline PANFILI (1993) & étang & France & $17-67$ & $\mathrm{Rp}$ & entier & 908 & 26,3 & 1,16 & 80 \\
\hline PANFILI (1993) & canal & France & $7-75$ & $R p$ & entier & 513 & 25,9 & 5,33 & 74 \\
\hline
\end{tabular}




\section{Courbes de croissance}

Sur le plan biologique, l'anguille européenne est un très bon modèle pour caractériser l'influence de l'environnement sur ses populations. En effet, à chaque génération (reproduction dans les Sargasses), les anguilles qui arrivent sur continent ont un pool génétique commun (YAHYAOUI, 1983) puis se répartissent ensuite dans les divers biotopes. Les différences de croissance seront donc essentiellement imputables à l'action de facteurs épigénétiques.

Les résultats de croissance montrent qu'il existe des différences importantes entre les biotopes colonisés par l'anguille (Tableau II et fig. 10). La variabilité de la croissance en milieu naturel est donc importante ; elle avait été soulignée dans plusieurs études (VOLLESTAD, 1989, 1992 ; FONTENELLE, 1991 ; PANFILI et al., 1994). L'influence du sexe sur la croissance n'est pas présentée dans le Tableau II, mais de nombreuses études ont montré la plus forte croissance des femelles en milieu naturel (TESCH, 1977 ; LEE, 1979 ; ROSSI, 1979 ; ROSSI et VILLANI, 1980 ; APRAHAMIAN, 1986, 1988 ; VOLLESTAD et JONSSON, 1988 ; PANFILI et al., 1994) et surtout en aquaculture (EGUSA, 1979). Les différences de croissance peuvent aussi être très marquées entre les individus d'un même milieu (PANFILI et al., 1994).

Le Tableau II se base sur des comparaisons de croissance interannuelles, ce qui est plus justifiable compte tenu des méthodes d'otolithométrie très variées. Les comparaisons ne se font plus directement sur des tailles à chaque âge sur les courbes de croissance. Les tailles à un an sont par ailleurs plus élevées si l'on tient compte des résultats obtenus par observation directe de la taille à un âge donné plutôt que des résultats rétrocalculés. Par exemple, pour deux estuaires espagnols, la taille à un an observée est de $25,3 \mathrm{~cm}$ pour ARIAS et DRAKE (1985) et la taille rétrocalculée par FERNANDEZ-DELGADO et al. (1989) est de $13,5 \mathrm{~cm}$. Le fait le plus caractéristique est la différence sensible entre les milieux "saumâtres" et les milieux "d'eau douce". Ceci est remarquable non seulement pour les tailles à un an, entre 10,2 et $25,3 \mathrm{~cm}$ pour l'eau saumâtre et entre 10,0 et $16,0 \mathrm{~cm}$ pour l'eau douce, mais aussi pour les accroissements interannuels (Tableau II). Dès le départ, les individus qui grandissent dans les eaux dulçaquicoles présentent un retard en taille par rapport à ceux qui fréquentent les sites proches de la mer, et cette différence sera ensuite accentuée par des taux de croissance plus faibles en eau douce. Les croissances dans les eaux saumâtres méditerranéennes semblent meilleures que partout ailleurs, jusqu'à $15,2 \mathrm{~cm}$ (FERNANDEZ-DELGADO et al., 1989), $14,5 \mathrm{~cm}$ (ROSSI et VILLANI, 1980), et $9,0 \mathrm{~cm}$ (PANFILI, 1993) entre la première et la deuxième année; ensuite les accroissements annuels sont variables avec une tendance à la diminution vers les classes âgées. Dans les milieux dulçaquicoles, on observe cependant des croissance importantes dans des régions pourtant septentrionales (VOLLESTAD et JONSSON, 1988 ; NAGIEC et BAHNSAWY, 1990). PANFILI (1993) ayant étudié et comparé deux populations saumâtre et dulçaquicole, dans deux régions méditerranéennes proches, avec un même outil de lecture d'âge, montre que la différence se fait surtout dès la première année, et qu'ensuite les taux de croissance sont comparables. II faut rattacher ces différences (1) aux périodes d'arrivée des civelles dans les biotopes, (2) à la disponibilité, la qualité et la quantité de nourriture disponible et à son turnover (les lagunes sont considérées comme des milieux riches où viennent se reproduire de nombreuses espèces), (3) à la compétition interspécifique.

\section{CONCLUSION}

Chez l'anguille européenne, la variabilité de la croissance est observée à différents niveaux : structures de croissance sur les otolithes, rythmes de leur dépôt, morphométrie des otolithes, croissance somatique entre les milieux ou à l'intérieur d'un même milieu. Les études de l'âge et de la croissance de cette espèce ne seront donc pas aisées. La notion d'écotỹpe oblige pratiquement à ne prendre en compte que la population étudiée, et à valider de façon rigoureuse les observations. Il est donc difficile de faire des comparaisons sur une grande échelle, les disparités de croissance étant très marquées et les méthodes utilisées souvent très différentes. Les milieux saumâtres représentent des sites de croissance plus favorables que les milieux dulçaquicoles. On peut alors se demander pourquoi les anguilles colonisent tous les milieux, même ceux où leur. croissance risque d'être moins bonne : une sélection du milieu avec un "homing" marqué est peut être impossible dans le cas de l'anguille où un brassage génétique se produit à chaque génération. 
Tableau II : Comparaison des croissances estimées à partir des otolithes d'anguilles européennes dans divers milieux par différents auteurs. Les croissances se présentent sous la forme d'accroissements (en $\mathrm{cm}$ ) entre deux classes d'âge voisines, calculés par observation directe de la longueur associée au groupe d'âge (obs.) ou par un rétrocalcul à partir des anneaux de croissance (rétroc.). Le classement ne tient pas compte de la chronologie des observations mais plutôt de la ressemblance des milieux.

Table II : Growth comparisons estimated from otoliths of European eels in different environments by different authors. The growth is indicated by the increment in length $(\mathrm{cm})$ between two successive age classes, calculated from the direct observation of lengths-at-age (obs.) or from back-calculated data (rétroc.). The presentation takes into account the similarities of environments more than the chronology of the publications.

\begin{tabular}{|c|c|c|c|c|c|c|c|c|c|c|c|c|}
\hline \multirow[b]{2}{*}{ auteurs } & \multirow[b]{2}{*}{ milieu } & \multirow[b]{2}{*}{ pays } & \multirow[b]{2}{*}{ méthode } & \multirow[b]{2}{*}{$\begin{array}{l}\text { taille } \\
\text { à } 1 \text { an }\end{array}$} & \multicolumn{8}{|c|}{ Accroissement entre deux classes d'âge $(\mathrm{cm})$} \\
\hline & & & & & $1-2$ & $2-3$ & $3-4$ & 4-5 & $5-6$ & $6-7$ & 7-8 & 8-9 \\
\hline PENAZ et TESCH (1970) & mer & Allemagne & rétroc. & 12,1 & 5,0 & 5,0 & 4,4 & 3,5 & - & - & - & - \\
\hline PENAZ et TESCH (1970) & estuaire & Allemagne & rétroc. & 11,9 & 3,7 & 4,1 & 4,5 & 5,1 & - & - & $\therefore$ & - \\
\hline LEE et LASSERRE (1970) & lagune & France & obs. & 20,1 & 7,0 & 7,1 & - & - & 6,9 & 1,5 & 3,2 & - \\
\hline ROSSI et VILANI (1980) & lagune & Italie & rétroc. & 22,7 & 14,5 & 6,7 & 1,6 & 12,8 & - & - & - & - \\
\hline $\begin{array}{l}\text { ARDIZZONE et } \\
\text { CORSI (1985) }\end{array}$ & lagune & Italie & obs. & 23,3 & 4,1 & 4,7 & - & - & - & $\cdot$ & $\therefore$ & - \\
\hline ARIAS et DRAKE (1985) & estuaire & Espagne & obs. & 25,3 & 6,2 & 6,7 & 6,8 & - & - & - & - & - \\
\hline LECOMTE-FINIGER (1985) & lagune & Frąnce & rétroc. & 10,2 & 7,2 & 7,6 & - & - & - & - & - & - \\
\hline VOLLESTAD (1986) & lagune & Norvège & - & 12,8 & 7,2 & 7,7 & 5,1 & 5,0 & 4,4 & 5,5 & 3,2 & 2,2 \\
\hline MALLAWA (1987) I & lagune & France & rétroc. & 15,5 & 6,1 & 5,4 & 4,0 & 4,3 & - & - & - & - \\
\hline MALLAWA (1987) M & lagune & France & rétroc. & 14,2 & 6,4 & 6,1 & 4,6 & 3,4 & 2,4 & 3,3 & - & - \\
\hline $\begin{array}{l}\text { MALLAWA (1987) F } \\
\text { FERNANDEZ-DELGADO }\end{array}$ & lagune & France & rétroc. & 14,4 & 7,1 & 6,5 & 5,6 & 5,4 & 5,1 & 5,1 & 6,3 & 6,6 \\
\hline et al. (1989) & estuaire & Espagne & rétroc. & 13,5 & 15,2 & 9,8 & 5,8 & 6,1 & 3,7 & - & - & - \\
\hline GORDO et JORGE (1991) & lagune & Portugal & obs. & 17,2 & 6,1 & 6,2 & 5,6 & 5,0 & 5,5 & 5,6 & 3,8 & 3,8 \\
\hline FONTENELLE (1991) & lat. $<45^{\circ}$ & {$[\mathrm{sal}]<25 \mathrm{~g} / 1$} & 2 & 24,3 & 7,7 & 3,9 & 1,2 & 3,0 & 2,2 & 4,0 & - & - \\
\hline $\begin{array}{l}\text { MALLAWA et } \\
\text { LECOMTE-FINIGER (1992) }\end{array}$ & lagune & France & obs. & 22,0 & 2,8 & 4,8 & 2,9 & 2,4 & - & $\cdot$ & - & - \\
\hline MOUNAIX (1992b) & estuaire & France & obs. & 12,6 & 7,2 & 4,5 & 5,9 & 16,2 & 15,5 & - & - & - \\
\hline MOUNAIX (1992b) & estuaire & France & rétroc. & 14,7 & 5,3 & 7,6 & 4,5 & 5,4 & 6,2 & - & - & $\cdot$ \\
\hline PANFILI (1993) & lagune & France & rétroc. & 19,5 & 9,0 & 6,1 & 6,4 & 7,0 & - & - & - & - \\
\hline PANFILI et al. (1994) & étang & France & rétroc. & - & 6,7 & 7,4 & 5,1 & 3,9 & 3,6 & - & - & - \\
\hline SINHA et JONES (1967) & rivière & Angleterre & obs. & 13,6 & 2,6 & 3,9 & 3,4 & 4,1 & 2,6 & $\cdot$ & - & $\cdot$ \\
\hline PENAZ et TESCH (1970) & rivière & Allemagne & rétroc. & 11,9 & 4,5 & 5,0 & 4,6 & 2,0 & - & - & - & - \\
\hline RASMUSSEN et & & & & & & & & & & & & \\
\hline THERKILDSEN (1979) & rivière & Danemark & obs. & 10,8 & 4,5 & 3,4 & 7,8 & 1,3 & 6,6 & 4,5 & 1,1 & 0,7 \\
\hline MORIARTY (1983) & rivière & Irlande & rétroc. & $\cdot$ & 4,8 & 4,2 & 3,7 & 3,6 & 3,4 & 3,2 & 3,2 & 3,1 \\
\hline BERG (1985) & lac & Allemagne & rétroc. & 11,9 & 5,1 & 5,4 & 5,4 & 4,8 & 5,2 & 4,4 & 4,0 & 3,6 \\
\hline PAULOVITZ et BIRO (1986) & lac & Hongrie & rétroc. & 13,4 & 9,4 & 9,5 & 7,1 & 5,4 & 5,0 & 5,1 & 4,8 & 5,6 \\
\hline $\begin{array}{l}\text { VOLLESTAD et } \\
\text { JONSSON (1988) }\end{array}$ & rivière & Norvège & rétroc. & 13,3 & 8,4 & 8,3 & 7,8 & 6,7 & 5,5 & 4,2 & 3,4 & 2,7 \\
\hline $\begin{array}{l}\text { NAGIEC et } \\
\text { BAHNSAWY (1990) }\end{array}$ & lac & Pologne & rétroc. & 13,0 & 6,5 & 4,8 & 3,9 & 4,6 & 3,9 & 3,9 & 3,6 & 3,6 \\
\hline $\begin{array}{l}\text { MANN et } \\
\text { BLACKBURN (1991) }\end{array}$ & torrent & Angleterre & obs. & 10,0 & 2,0 & 3,5 & 2,0 & 3,5 & 4,0 & - & $\cdot$ & - \\
\hline POOLE (1991) & torrent & Irlande & rétroc. & - & 1,7 & 1,5 & 1,5 & 1,3 & 1,3 & 1,2 & 1,3 & 1,2 \\
\hline MOUNAIX (1992b) & fleuve & France & obs. & 14,0 & 9,5 & 6,1 & 1,0 & 2,9 & 11,6 & - & - & - \\
\hline MOUNAIX (1992b) & fleuve & France & rétroc. & 16,0 & 4,0 & 2,5 & 2,5 & 1,0 & 12,5 & - & $\cdot$ & - \\
\hline PANFILI (1993) & canal & France & rétroc. & 12,4 & 6,2 & 6,3 & 6,0 & 5,4 & 4,0 & 4,0 & 4,4 & 4,2 \\
\hline
\end{tabular}




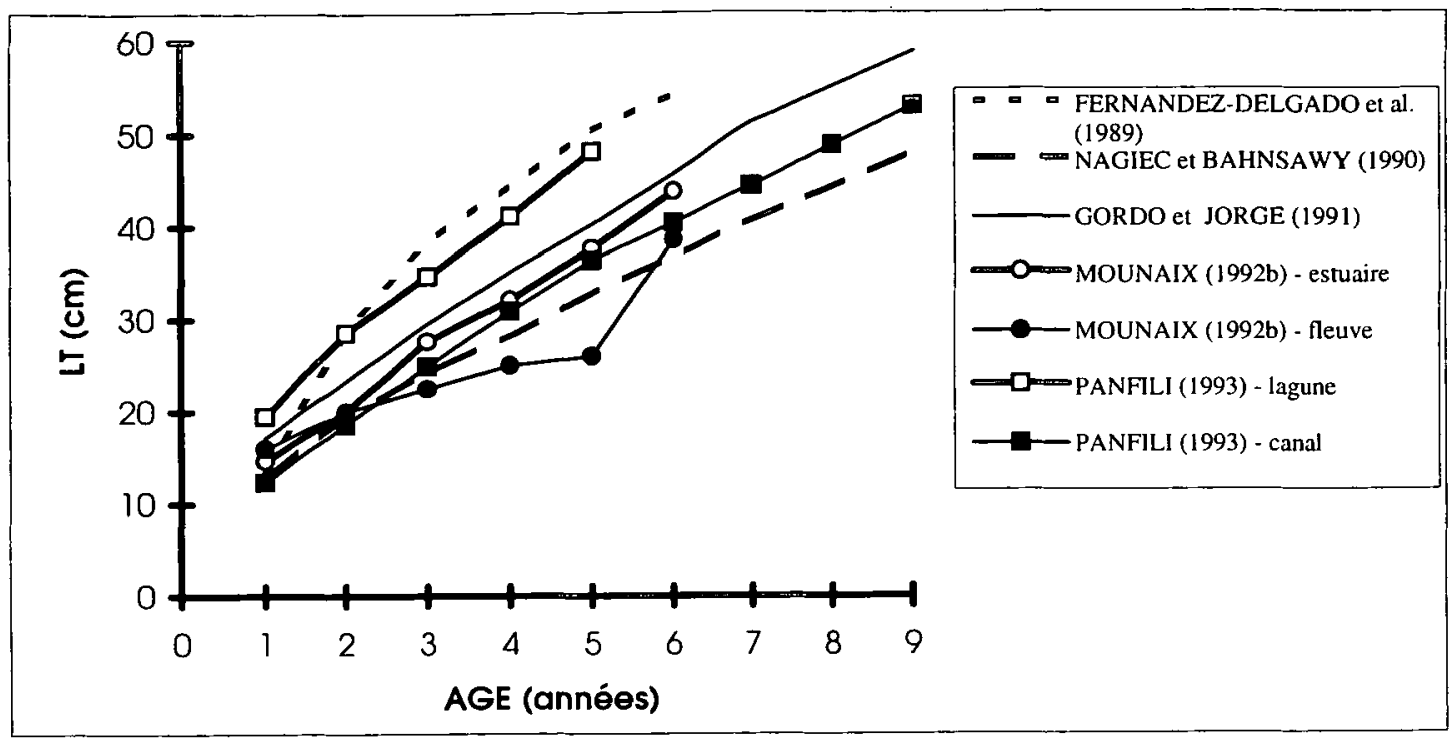

Figure 10 : Courbes de croissance de diverses populations de l'anguille européenne d'après des données rétrocalculées de la littérature.

Figure 10 : Growth curves of some European eel populations from back-calculated data of the literature.

\section{BIBLIOGRAPHIE}

ALBRECHTSEN K., 1968. A dyeing technique for otolith age reading. J. Cons. Perm. int. Explor. Mer, 32, 278-280.

APRAHAMIAN M.W., 1986. Eel (Anguilla anguilla L.) production in the River Severn, England. Polskie Arch. Hydrobiol., 33 (3/4), 373-389.

APRAHAMIAN M.W., 1987. Use of the burning technique for age determination in eels (Anguilla anguilla (L.)) derived from the stocking of elvers. Fish. Res., 6, 93-96.

APRAHAMIAN M.W., 1988. Age structure of eel, Anguilla anguilla (L.), populations in the River Severn, England, and the River Dee, Wales. Aquacult. Fish. Manag., 19, 365-376.

ARDIZZONE G.D., CORSI F., 1985. Eel population structure, dynamics and fishing yield in a Mediterranean coastal lagoon. OEbalia, 11 (2), 547-560.

ARIAS A.M., DRAKE P., 1985. Estructura de la población y régimen alimentario de Anguilla anguilla L., 1758 (Osteichthyes, Anguillidae), en los esteros de San Fernando (Cádiz). Invest. Pesq., 49-(4), 475-491.

BAGLINIĖRE J.L., CASTANET J., CONAND F., MEUNIER F.J., 1992. Terminologie en sclérochronologie chez les Vertébrés in BAGLINIËRE J.L., CASTANET J., CONAND F., MEUNIER F.J., Tissus durs et âge individuel des Vertébrés, Colloque National, Bondy, France, 4-6 mars 1991, 443-447, Colloque et Séminaires ORSTOM-INRA, Paris.

BEAMISH R.J., MCFARLANE G.A., 1983. The forgotten requirement for age validation in fisheries biology. Trans. Am. Fish. Soc., 112, 735-743.

BEAMISH R.J., MCFARLANE G.A., 1987. Current trends in age determination methodology in SUMMERFELT R.C., HALL G.E., The age and growth of fish, 15-42, The lowa State University Press, Ames, lowa. 
BENECH V., 1975. Note sur la préparation des otolithes, plus particulièrement ceux de l'anguille. Ann. Hydrobiol., 6 (2), 173-178.

BERG R., 1985. Age determination of eels, Anguilla anguilla (L.), comparison of field data with otolith ring patterns. J. Fish Biol., 26, 537-544.

BOETIUS I., 1985. Otoliths of eels of known age. EIFAC Working Party on Eels, Perpignan, September $17-18,5 \mathrm{p}$.

BOETIUS I., 1986. Otoliths of eels of known age. Vie Milieu, 36 (4), 298.

CAMPANA S.E., 1990. How reliable are growth back-calculations based on otoliths ? Can. J. Fish. Aquat. Sci., 47, 2219-2227.

CASTONGUAY M., SIMARD P., GAGNON P., 1991. Usefulness of Fourier analysis of otolith shape for Atlantic mackerel (Scomber scombrus) stock discrimination. Can. J. Fish. Aquat. Sci., 48, 296-302.

CHARLON N., 1975. Technique de préparation des otolithes. EIFAC Montpellier, 12-17 Mai, 2 p.

CHISNALL B.L., HAYES J.W., 1991. Age and growth of shortfinned eels (Anguilla australis) in the lower Waikato basin, North Island, New Zealand. N-Z. J. Mar. Freshwater Res., 25, 71-80.

DAWSON W.A., 1986. Mackerel (Scomber scombrus L.) otolith L1 analysis as a method of stock separation. ICES, Pelagic Fish Com., CM 1986/H:24, 7 p.

DEELDER C.L., 1976. The problem of the supernumerary zones in otoliths of the European eel (Anguilla anguilla, Linnaeus 1758); a suggestion to cope with it. Aquaculture, 9, 373-379.

DEELDER C.L., 1981. On the age and growth of cultured eels, Anguilla anguilla (Linnaeus, 1758). Aquaculture, 26, 13-22.

DEKKER W., 1986. Age reading of European eels using tetracycline labelled otoliths. Int. Coun. Explor. Sea, Anadrom. Catadrom. Fish Comm., C.M.1986/M:16, 14 p.

DEKKER W., 1987. Further results on age reading of European eel using tetracyclin labeled otoliths. EIFAC Working Party on Eel, Bristol, $5 \mathrm{p}$.

EGUSA S., 1979. Notes on the culture of the European eel (Anguilla anguilla L.) in Japanese farming pond. Rapp. P.-v. Réun. Cons. int. Explor. Mer, 174, 51-58.

FERNANDEZ-DELGADO C., HERNANDO J.A., HERRERA M., BELLIDO M., 1989. Age and growth of yellows eels, Anguilla anguilla, in the estuary of the Guadalquivir river (South-West Spain). J. Fish Biol., 34, 561-570.

FINIGER R., 1976. Contribution à l'étude biologique et écologique des civelles (Anguilla anguilla Linné, 1758) lors de leur pénétration dans un étang méditerranéen. I. Recrutement et biométrie au cours d'un cycle annuel. Vie Milieu, 26 (1A), 123-144.

FONTENELLE G., 1991. Age et longueur moyenne des anguilles (Anguilla anguilla) en Europe : une revue critique. EIFAC Working Party on Eel, Dublin, $16 \mathrm{p}$.

FRANCIS R.I.C.C., 1990. Back-calculation of fish length : a critical review. J. Fish Biol., 36, 883-902.

FRIEDLAND K.D., REDDIN D.G., 1994. Use of otolith morphology in stock discriminations of Atlantic salmon (Salmo salar). Can. J. Fish. Aquat. Sci., 51, 91-98.

GORDO L.S., JORGE M.I., 1991. Age and growth of the European eel, Anguilla anguilla (Linnaeus, 1758), in the Aveiro lagoon, Portugal. Sci. Mar., 55 (2), 389-395.

HANSEN R.A., EVERSOLE A.G., 1984. Age, growth, and sex ratio of American eels in brackish-water portions of a South Carolina river. Trans. Am. Fish. Soc., 113, 744-749.

HARALDSTAD O., 1984. Albersbestemmelse av al Anguilla anguilla. Fauna, 37, 71-75. 
HETCH T., APPELBAUM S., 1977. Otolith length/fish length relationship of leptocephali, elvers and sub-adults (reared) eels (Anguilla anguilla). ICES, CM 1977/M:4, 9 p.

HOPKINS P.J., 1986. Mackerel stock discrimination using otolith morphometrics. ICES, CM 1986/H:7, 5 p.

HU L.C., TODD P.R., 1981. An improved technique for preparing eel otoliths for aging. $N-Z$. J. Mar. Freshwater Res., 15, 445-446.

JELLYMAN D.J., 1979. Scale development and age determination in New Zealand freshwater eels (Anguilla spp.). N-Z. J. Mar. Freshwater Res., 13 (1), 23-30.

KUHLMANN H., 1976. Influence of temperature, food, size and origin on the growth and sexual differenciation of elvers (Anguilla anguilla). ICES / EIFAC Symposium on Eel Research and Management, 12,17 $\mathrm{p}$.

KUHLMANN H., 1979. The influence of temperature, food, initial size, and origin of elvers (Anguilla anguilla L.). Rapp. P.-v. Cons. int. Explor. Mer, 174, 59-63.

LECOMTE-FINIGER R., 1985. L'âge de l'anguille européenne (Anguilla anguilla L., 1758), état actuel des connaissances et recherches nouvelles en Méditerranée. J. applied Ichthyol., 4, 178-192.

LECOMTE-FINIGER R., 1992a. The crystalline ultrastructure of otoliths of the eel $(A$. anguilla L. 1758). J. Fish Biol., 40, 181-190.

LECOMTE-FINIGER R., 1992b. Situation actuelle des méthodes d'évaluation de l'âge de l'anguille, Anguilla anguilla, in BAGLINIÈRE J.L., CASTANET J., CONAND F., MEUNIER F.J., Tissus durs et âge individuel des Vertébrés, Colloque National, Bondy, France, 4-6 mars 1991, 281-297, Colloque et Séminaires ORSTOM-INRA, Paris.

LECOMTE-FINIGER R., YAHYAOUI A., 1989. La microstructure de l'otolithe au service de la connaissance du développement larvaire de l'anguille européenne, Anguilla anguilla. C. R. Acad. Sci. Paris, 308 (Ser.III), 1-7.

LEE T.W., 1979. Dynamique des populations d'anguilles (Anguilla anguilla L.) des lagunes du Bassin d'Arcachon. Thèse 3ème Cycle, Univ. Montpellier, France, $218 \mathrm{p}$.

LEE T.W., LASSERRE G., 1979. Analyse de la structure et estimation du stock d'une population d'anguilles d'un réservoir à poisson du bassin d'Arcachon. Bull. Ecol., 10 (2), 139-145.

LIEW P.K.L., 1974. Age determination of American eels based on the structure of their otoliths in BAGENAL T.B., The ageing of fish, 124-136, Unwin Brother's Ltd, London.

MACEINA M.J., MURPHY B.R., 1989. Differences in otolith morphology among the two subspecies of largemouth bass and their F1 hybrid. Trans. Am. Fish. Soc., 118, 573-575.

MACEWAN A., HECHT T., 1984. Age and growth of the longfin eel, Anguilla mossambica Peters, 1852 (Pisces : Anguillidae) in Transkei rivers. S. Afr. J. Zool., 19 (4), 280-285.

MALLAWA A., 1987. Dynamique des stocks exploités et halieutiques de l'anguille européenne ( $A$. anguilla $L$. 1758) des lagunes du Narbonnais et du Roussillon (Bages-Sigean et Canet-Saint-Nazaire), Golfe du Lion. Thèse 3ème Cycle, Univ. Perpignan, France, $406 \mathrm{p}$.

MALLAWA A., LECOMTE-FINIGER R., 1992. Comparative study of two populations of Anguilla anguilla (Linnaeus, 1758) eels from French Mediterranean lagoons (BagesSigean and Canet-Saint-Nazaire) : age population and structure. Sci. Mar., 56 (1), 1-6.

MANN R.H.K., BLACKBURN J.H., 1991. The biology of the eel Anguilla anguilla (L.) in an English chalk stream and interactions with juvenile trout Salmo trutta $L$. and salmon Salmo salar L. Hydrobiologia, 218, 65-76.

MESSIEH S.N., MACDOUGALL C., CLAYTOR R., 1989. Separation of Atlantic herring (Clupea harengus) stocks in the Southern Gulf of St. Lawrence using digitized otolith morphometrics and discriminant function analysis. Can. Tech. Rep. Fish. Aquat. Sci., $1647,22 \mathrm{p}$. 
MEUNIER F., 1974. La technique de marquage vital des tissus squelettiques des poissons. Bull. Fr. Piscic., 255, 51-57.

MEUNIER F.J., BOIVIN G., 1974. Divers aspects de la fixation du chlorhydrate de tétracycline sur les tissus squelettiques de quelques Téléostéens. Bull. Soc. Zool. Fr., 99 (3), 495-504.

MICHAUD M., DUTIL J.D., DODSON J.J., 1988. Determination of the age of young American eels, Anguilla rostrata, in fresh water, based on otolith surface area and microstructure. J. Fish Biol., 32, 179-189.

MORIARTY C., 1973. A technique for examining eel otoliths. J. Fish Biol., .5, 183-184.

MORIARTY C., 1983. Age determination and growth rate of eels, Anguilla anguilla (L.). J. Fish Biol., 23, 257-264.

MORIARTY C., STEINMETZ B., 1979. On age determination of eel. Rapp. P.-v. Réun. Cons. int. Explor. Mer, 174, 70-74.

MOUNAIX B., 1991. Utilisation des otolithes pour caractériser l'habitat de l'anguille jaune dans le bassin de la Vilaine, Bretagne. EIFAC Working Party on Eel, Dublin, 11 p.

MOUNAIX B., 1992a. Validation de l'estimation de l'âge de l'anguille européenne, Anguilla anguilla, dans le bassin versant de la Vilaine (Bretagne) : résultats préliminaires in BAGLINIERE J.L., CASTANET J., CONAND F., MEUNIER F.J., Tissus durs et âge individuel des Vertébrés, Colloque National, Bondy, France, 4-6 mars 1991, 109-117, Colloque et Séminaires ORSTOM-INRA, Paris.

MOUNAIX B., 1992b. Intercalibration et validation des méthodes d'estimation de l'âge de l'anguille européenne (Anguilla anguilla) : application au bassin versant de la Vilaine, Bretagne. Thèse Doctorat, ENSA Rennes, France, $146 \mathrm{p}$.

NAGIEC M., BAHNSAWY M.H., 1990. Age and growth of female eels, Anguilla anguilla L., in a Polish lake, Jeziorak lake, Mazurian lake district, Poland. Aquacult. Fish. Manage., 21, 459-470.

PANFILI J., 1993. Estimation de l'âge individuel des poissons : méthodologies et applications à des populations naturelles tropicales et tempérées. ORSTOM Paris Trav. Doc. Microfich., $456 \mathrm{p}+$ ann.

PANFILI J., XIMÉNÈS M.C., DO CHI T., 1990. Age determination of eels in the French Mediterranean lagoons using classical methods and an image analysis system. Int. Rev. gesam. Hydrobiol., 75 (6), 745-754.

PANFILI J., XIMÉNĖS M.C., CRIVELLI A.J., DO CHI T., 1992. Validation de l'âge de l'anguille européenne dans les lagunes méditerranéennes françaises (Camargue) : résultats préliminaires in BAGLINIERE J.L., CASTANET J., CONAND F., MEUNIER F.J., Tissus durs et âge individuel des Vertébrés, Colloque National, Bondy, France, 4-6 mars 1991, 119-127, Colloque et Séminaires ORSTOM-INRA, Paris.

PANFILI J., XIMÉNĖS M.C., CRIVELLI A.J., 1994. Sources of variation in growth of the European eel (Anguilla anguilla) estimated from otoliths. Can. J. Fish. Aquat. Sci, 51, 506-515.

PAULOVITS G., BIRO P., 1986. Age determination and growth of eel, Anguilla anguilla (L.), in Lake Ferto, Hungary. Fish. Res., 4 (2), 101-110.

PENAZ V.M., TESCH F.W., 1970. Geschlechtsverhältnis und wachstum beim aal an verschiedenen lokalitäten von Nordsee und Elbe. Ber. Dt. Wiss. Komm. Meeresforsch, 21, 290-310.

POOLE R., 1991. The age and growth of eels (Anguilla anguilla L.) in two oligotrophic streams in the west of Ireland. EIFAC Working Party on Eels, Dublin, $12 \mathrm{p}$.

RASMUSSEN G., THERKILDSEN B., 1979. Food, growth, and production of Anguilla anguilla L. in a small Danish stream. Rapp. P.-v. Réun. Cons. int. Explor. Mer, 174, 32-40. 
RICKER W.E., 1992. Back-calculation of fish lengths based on proportionality between scale and length increments. Can. J. Fish. Aquat. Sci., 49, 1018-1026.

ROSSI R., 1979. An estimate of the production of the eel population in the Valli of Comacchio (Po Delta) during 1974-1976. Boll. Zool., 46, 217-223.

ROSSI R., VILLANI P., 1980. A biological analysis of eel catches, Anguilla anguilla L., from the lagoons of Lesina and Varano, Italy. J. Fish Biol., 16, 413-423.

SADLER K., 1979. Effects of temperature on the growth and survival of the European eel, Anguilla anguilla L. J. Fish Biol., 15, 499-507.

SINHA V.R.P., JONES J.W., 1967. On the age and growth of freshwater eel (Anguilla anguilla). J. Zool. London, 153, 99-117.

SMALL M.A., TAYLOR W.W., 1987. Sources of back-calculation error in estimating growth of lake whitefish in SUMMERFELT R.C., HALL G.E., The age and growth of fish, 189-202, The lowa State Univ. Press, Ames, lowa, USA.

TESCH F.W., 1977. The eel biology and management of anguillid eels. Chapman \& Hall, $435 \mathrm{p}$.

VÉRO M., PAULOVITS G., BIRO P., 1986. An improved grinding technique for examining fish otoliths for age and growth studies with special consideration of the eel Anguilla anguilla L. Aquacult. Fish. Manage., 17 (3), 207-212.

VOLLESTAD L.A., 1985. Age determination and growth of yellow eels, Anguilla anguilla (L.), from a brackish water, Norway. J. Fish Biol., 26, 521-525.

VOLLESTAD L.A., 1986. Growth and production of female yellow eels (Anguilla anguilla L.) from brackish water in Norway. Vie Milieu, 36 (4), 267-271.

VOLLESTAD L.A., 1989. Effect of growth rate on age at maturity of the European eel Anguilla anguilla. EIFAC Working Party on Eel, Porto, Portugal, $22 \mathrm{p}$.

VOLLESTAD L.A., 1992. The geographic variation in age and length at metamorphosis of maturing European eel : environmental effects and phenotypic plasticity. J. Anim. Ecol., 61, 41-68.

VOLLESTAD L.A., JONSSON B., 1988. A 13-year study of the population dynamics and growth of the European eel Anguilla anguilla in a Norvegian river : evidence for density-dependent mortality, and development of a model for predicting yield. $J$. Anim. Ecol., 57, 983-997.

VOLLESTAD L.A., LECOMTE-FINIGER R., STEINMETZ B., 1988. Age determination of Anguilla anguilla and related species. EIFAC Occas. Pap., 21, 1-28.

VOLLESTAD L.A., NAESJE T.F., 1988. Reading otoliths of eels Anguilla anguilla (L.) of known age from Kolderveen, The Netherlands. Aquacult. Fish. Manage., 19, 387-391.

WEBER D., RIGWAY G.J., 1967. Marking Pacific salmon with tetracycline antibiotics. J. Fish. Res. Board Can., 24 (4), 849-865.

XIMÉNĖS M.C., 1986. L'anguille en Méditerranée française : aspects écobiologiques et halieutiques. Rapp. Ministère Mer, CEMAGREF Montpellier-ALA, France, 166 p.

YAHYAOUI A., 1983. Etude comparée (recrutement, croissance et polymorphisme enzymatique) des populations atlantiques et méditerranéennes (Maroc-France) des civelles d'Anguilla anguilla L., 1758. Thèse 3ème Cycle, Univ. Perpignan, France, 176 p. 